\title{
Spin-Mechanics with Nitrogen-Vacancy Centers and Trapped Particles
}

\author{
Maxime Perdriat ${ }^{1}$, Clément Pellet-Mary ${ }^{1}$, Paul Huillery ${ }^{1}$, Loïc Rondin ${ }^{2}$ iD and Gabriel Hétet ${ }^{1, *}$ \\ 1 Laboratoire De Physique de l'École Normale Supérieure, École Normale Supérieure, PSL Research University, \\ CNRS, Sorbonne Université, Université de Paris, 24 rue Lhomond, CEDEX 05, 75231 Paris, France; \\ maxime.perdriat@phys.ens.fr (M.P.); clement.pellet-mary@phys.ens.fr (C.P.-M.); \\ paul.huillery@gmail.com (P.H.) \\ 2 Université Paris-Saclay, CNRS, ENS Paris-Saclay, Centrale-Supélec, LuMIn, 91190 Gif-sur-Yvette, France; \\ loic.rondin@universite-paris-saclay.fr \\ * Correspondence: gabriel.hetet@ens.fr
}

Citation: Perdriat, M.; Pellet-Mary, C.; Huillery, P.; Rondin, L.; Hétet, G. Spin-Mechanics with NitrogenVacancy Centers and Trapped Particles. Micromachines 2021, 12, 651. https://doi.org/10.3390/mi12060651

Academic Editors: Elke Neu and Štěpán Potocký

Received: 19 April 2021

Accepted: 26 May 2021

Published: 1 June 2021

Publisher's Note: MDPI stays neutral with regard to jurisdictional claims in published maps and institutional affiliations.

Copyright: (C) 2021 by the authors Licensee MDPI, Basel, Switzerland. This article is an open access article distributed under the terms and conditions of the Creative Commons Attribution (CC BY) license (https:// creativecommons.org/licenses/by/ $4.0 /)$.

\begin{abstract}
Controlling the motion of macroscopic oscillators in the quantum regime has been the subject of intense research in recent decades. In this direction, opto-mechanical systems, where the motion of micro-objects is strongly coupled with laser light radiation pressure, have had tremendous success. In particular, the motion of levitating objects can be manipulated at the quantum level thanks to their very high isolation from the environment under ultra-low vacuum conditions. To enter the quantum regime, schemes using single long-lived atomic spins, such as the electronic spin of nitrogen-vacancy (NV) centers in diamond, coupled with levitating mechanical oscillators have been proposed. At the single spin level, they offer the formidable prospect of transferring the spins inherent quantum nature to the oscillators, with foreseeable far-reaching implications in quantum sensing and tests of quantum mechanics. Adding the spin degrees of freedom to the experimentalists toolbox would enable access to a very rich playground at the crossroads between condensed matter and atomic physics. We review recent experimental work in the field of spin-mechanics that employ the interaction between trapped particles and electronic spins in the solid state and discuss the challenges ahead. Our focus is on the theoretical background close to the current experiments, as well as on the experimental limits, that, once overcome, will enable these systems to unleash their full potential.
\end{abstract}

Keywords: nitrogen-vacancy centers; micro-mechanical oscillators; electronic spin resonance

\section{Introduction}

The unique control offered by single quantum systems, such as atoms or ions, has enabled an immense boost in the development of quantum technologies. Extending these technologies to larger masses is important both for fundamental questions on the nature of quantum physics at larger scales, and for the development of innovative devices such as ultra-high precision force sensors and accelerometers [1].

Electro-, magneto- or opto- mechanically controlled levitating objects in vacuum are fascinating in this regard and have been at the focus of intense recent research activity [2]. This attention is supported by the exquisite control that one can exert over the levitated objects. These platforms indeed allow addressing of numerous degrees of freedom, easy tuning of the trapping potential, as well as enabling free-fall experiments [3], as in atomic physics.

Initial work proposed to coupling levitated silica nano-spheres, and even viruses, to the optical modes of a high finesse cavities [4-6]. The promises of this schemes have been supported by recent experiments that reported trapped particles cooled to the quantum regime [7-10]. Active development of force sensors are in progress and tests of various models of fundamental physics have also been proposed using various platforms [11-13]. To push these developments further and to enable operate the mechanical oscillator in the quantum 
regime, coupling the dynamics of the levitated system to a single intrinsically quantum system, such as ions, atoms, or artificial atoms, has been envisioned [14,15]. Towards this goal, amongst all condensed matter system, the negatively charged nitrogen-vacancy center $\left(\mathrm{NV}^{-}\right.$center for short) in diamond centers stands out because of the ease with which one can optically polarize and read-out its electronic spin under ambient conditions. Most of the proposals initially designed for clamped oscillators coupled with $\mathrm{NV}^{-}$centers $[14,16,17]$ can then be carried over to trapped diamonds [18] which are, in all current experiments, operating close to room temperature. Ensembles of $\mathrm{NV}^{-}$centers coupled identically to mechanical oscillators can also exhibit magnetic phase transitions, paving the way towards nano-scale magnetism with long-lived and controllable spins in a trapped particle $[19,20]$.

This growing research field will drive advances in quantum metrology via quantum enhanced gyroscopy and matter-wave interferometry $[19,21,22]$. The spin-mechanical coupling also brings important advantages for quantum sensing and metrology by providing enhanced measurement sensitivity. At the single spin level it also features additional non-linearity and/or control that could be helpful to build non classical states of motion [18]. Finally, they could also serve as transducers between optical and RF signals via the mechanical mode [23].

In this review, we describe the state-of-the-art levitation systems that involve $\mathrm{NV}^{-}$ centers and describe their specificities and limitations. The goal is not to draw a comparison between the performance of existing systems. Instead, we focus on the important results that have been accomplished and the remaining hurdles on the way towards operating in the quantum regime with these platforms.

In Section 2, we briefly present the existing levitation platform and the equations of motion describing the different mechanical modes. In Section 3, we present a rapid overview of the ancillary quantum system used to interact with the mechanical modes, namely the nitrogenvacancy center in diamond. The Hamiltonian of the total coupled spin-mechanical system is derived in Section 4. Section 5 reviews experiments that demonstrate read-out of the mechanical motion of levitated particles using the NV spin. Sections 6 and 7 provide a classical analytical treatment of spin-bistability, spin-spring and spin-cooling in the adiabatic limit. Finally, Section 8 presents the current challenges in spin-mechanics with trapped particles.

\section{Trapping Crystals}

The basic idea behind particle levitation is to hold a particle under atmospheric conditions or in vacuum against gravity. Here, after presenting the classical theoretical framework for harmonic motion analysis, we discuss current methods for trapping crystals in vacuum.

\subsection{Center of Mass Harmonic Motion}

The center of mass dynamics of a levitated particle along a direction parametrized by the coordinate $q$, can be described by a Langevin equation. For a stably trapped particle, one can linearize the trapping force so that the particle dynamics can, to a good approximation, be described by the equation of a harmonic oscillator

$$
m \frac{\mathrm{d}^{2} q}{\mathrm{~d} t^{2}}+m \gamma_{q} \frac{\mathrm{d} q}{\mathrm{~d} t}+m \omega_{q}^{2} q=F_{\mathrm{L}}(t)
$$

where $\gamma_{q}$ is the translational damping rate due to collisions with gas molecules and $F_{\mathrm{L}}$ is the Langevin fluctuating force induced by the interaction between the particle and the gas molecules, $m$ is the particle mass, and $\omega_{q}$ is the trapping frequency. Rarefied gas can be described as a Markovian thermal bath with a white noise spectrum so that the fluctuating force satisfies $\left\langle F_{\mathrm{L}}\right\rangle=0$ and $\left\langle F_{\mathrm{L}}(t) F_{\mathrm{L}}\left(t^{\prime}\right)\right\rangle=2 m \gamma_{q} k T \delta\left(t-t^{\prime}\right)$ at temperatures such that $k T \gg \hbar \omega_{q}$.

$\gamma_{q}$ depends on the exact shape of the particle [24] and is proportional to the residual gas pressure at low pressures. This property makes levitating platforms attractive, since the thermal noise can be made arbitrary small by reducing the gas pressure inside the vacuum 
chamber. Note that in practice, the dynamics of the particle may appear more complex than a simple harmonic oscillator at high temperatures because of instabilities induced by the non-linearity of the potential [25].

\subsection{Angular Confinement: The Librational Mode}

When considering the angular degree of freedom, two characteristic motional behaviours can be observed: pure rotation and libration, namely oscillation of the particle angle about a mean angular position. We will see that confining the angle so that the libration can be described by a harmonic oscillator is also of crucial importance when dealing with $\mathrm{NV}^{-}$ centers. The condition for librational confinement is that the total energy in the angular mode must not be larger than the angular potential depth. Typically, angular potentials are $\pi$ or $\pi / 2$-periodic so that when the standard deviation of the angle is in that range, the particle angle may jump from one angular well to another. This can be due to collisions with gas molecules for instance.

Once confined close to an angle $\theta=0$, the angle follows this equation of motion

$$
I \frac{d^{2} \theta}{d t^{2}}+I \gamma_{\theta} \frac{d \theta}{d t}+I \omega_{\theta}^{2} \theta=\tau_{L}(t) .
$$

$\tau_{L}(t)$ are Langevin fluctuation torques satisfying $\left\langle\tau_{\mathrm{L}}\right\rangle=0$ and $\left\langle\tau_{\mathrm{L}}(t) \tau_{\mathrm{L}}\left(t^{\prime}\right)\right\rangle=2 \gamma_{\theta} \operatorname{IkT} \delta(t-$ $\left.t^{\prime}\right) . \gamma_{\theta}$ is the damping rate for the angular degree of freedom due to collisions with gas molecules and $I$ the particle moment of inertia. As for translational modes, the damping rate for libration is proportional to the gas pressure at low pressures and strongly depends on the particle shape. According to the fluctuation dissipation theorem, the standard deviation of the angle $\theta$ is $\sqrt{\left\langle\theta^{2}\right\rangle}=\sqrt{k T /\left(I \omega_{\theta}^{2}\right)}$. One can thus obtain an approximate criterion for stable angular confinement requiring that $\sqrt{\left\langle\theta^{2}\right\rangle}$ is bounded by $\approx \pi / 10$ in order to ensure small angular deviations. We then obtain a condition on the angular rigidity $K_{t}=I \omega_{\theta}^{2}$ such that $K_{t} \gtrsim 10 \times k T$. Below this value, libration is not guaranteed, and angular deviations may be too large for efficient control of the NV electronic spin.

\subsection{Trapping Platforms}

The original proposals for trapping particles suggested use of optical forces. Under laser illumination, small dielectric particles can indeed become polarized. The induced dipole is then attracted to the highest intensity region where particles are stably trapped in three dimensions [26], with typical trapping frequencies in the $100 \mathrm{kHz}$ to $1 \mathrm{MHz}$ range. Reference [27] presents a broad overview of optical tweezers. Particles can be trapped and cooled either at the focus of a laser beam as in [28] (Figure 1a), at the node of a cavity field [29] or in the near-field of a photonic crystal [30]. Recent results in opto-mechanics with optical tweezers and cavities are described in a recent review [2].

Another means to trap particles, described in Figure 1b, is to use a Paul trap [31]. The radio-frequency modulation of a high voltage electric field applied to the trap electrode ensures three-dimensional confinement of charged particles with typical frequencies ranging from $100 \mathrm{~Hz}$ to $10 \mathrm{kHz}$. The center of mass motion of silica nano-particles [32], graphene flakes [33] and nanodiamonds [34] in Paul traps have been cooled to low temperatures under ultra-high vacuum levels using parametric feedback cooling.

Another way to trap micro-particles is to use magnetic fields. In magnetic traps, a magnetic object is levitating above a diamagnetic/superconductor material [35]. Alternatively, a diamagnetic particle - such as diamond — is levitating above magnets (see Figure 1c). Trapping frequencies in the hundreds of hertz range are typically observed. The latter trapping approach was efficiently employed to demonstrate cooling of the center of mass motion of trapped diamonds using feedback cooling [36,37]. 
(a) Optical tweezer

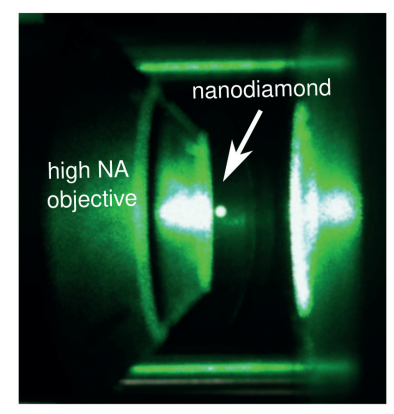

(b) Electrodynamic trap

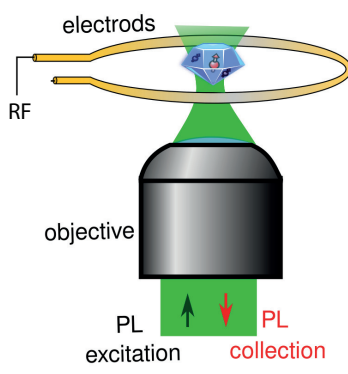

(c)

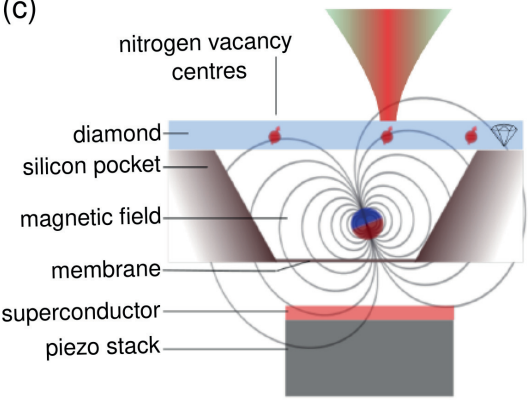

(d)

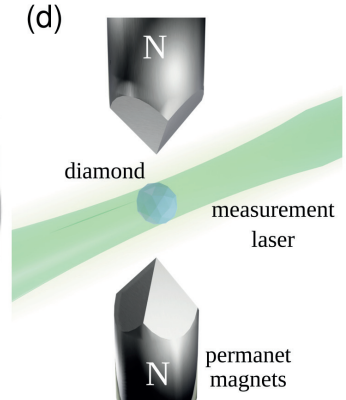

Figure 1. Different approaches to particle levitation. (a) Optical tweezers. Picture of an infrared laser focused through a high numerical aperture (NA) objective (shown on the left) allowing trapping a nanodiamond at its focus. The efficient scattering of the green laser used for NV excitation enables one to see the nanodiamond with the naked eye. (b) Paul trap: a charged diamond particle is held in vacuum by electric field gradients. (c) A magnetic particle is levitated above a superconductor. $\mathrm{NV}^{-}$centers implanted in a diamond slab above the levitated particle can be coupled with its motion through the magnetic field gradient. Adapted from ref. [35]. (d) A diamond particle is trapped by large magnetic field gradients thanks to its diamagnetism.

As already discussed, the other important degree of freedom for NV spin-mechanics is the particle angle. Trailblazing experiments with particles in optical tweezers have shown controlled rotation of silica spheres [38-41]. Very fast rotation frequencies up to a few $\mathrm{GHz}$ have also recently been observed using dumbbell-shaped silica nanoparticles under ultrahigh vacuum $[39,41]$. When using nanodiamonds, such rotational dynamics could open the door to observations of the Barnett effect on long-lived electronic spins [42] and to coupling spins to the gyroscopically stabilized angular motion. Importantly, stable angular confinement and the resulting librational motion of micro-particles was observed recently by several groups [43-45]. Angular confinement can be the result of the shape anisotropy and birefringence of the particles [43], or asymmetries of both charged particles and traps [46]. For micro-crystals in Paul traps, typical librational frequencies are in the $\mathrm{kHz}$ range. As discussed in Section 2.3, the moment of inertia must thus be larger than $\approx 10^{-27} \mathrm{~N} \cdot \mathrm{m}$ for Brownian motion not to make trapping unstable at $300 \mathrm{~K}$. Assuming a 1:2 ellipsoidal particle aspect ratio implies operation with particles with a greater axis diameter $d \gtrsim 1 \mu \mathrm{m}$. In optical traps, trapping frequencies can be as large as $1 \mathrm{MHz}$, implying that particles must have a moment of inertia larger than $10^{-33} \mathrm{~N} \cdot \mathrm{m}$. Particles with $d \gtrsim 50 \mathrm{~nm}$ must thus be employed to harmonically confine the angle at room temperature. The upper bound to the particle size will ultimately be limited by gravity, and by the specificities of the employed trapping mechanism. Observing such libration on small particles is an important step forward, because it will facilitate torque sensing with individual spins (see Section 6).

\section{Coupling to an Ancillary Quantum System: The Special Case of the $\mathbf{N V}^{-}$Center}

As discussed in the introduction, coupling a levitated particle to an ancillary quantum system is an exiting way to extend its capabilities. Coupling the internal electronic state of atoms or ions to trapped mechanical oscillators is intensively studied [47,48] and was successfully demonstrated with clamped oscillators [15,49,50]. Colloidal quantum dots [51], rare-earth ions in a solid matrix [52], or color centres in semi-conductor particles [53-55] have all been levitated in optical tweezers. These objects feature defects that behave as atoms which are hosted in a solid-state matrix, so these experiments are important steps forward. One important extra criterium is the coupling strength between the ancillary systems' quantum state and the levitated particle dynamics. The coupling strength quantifies how much the motion of the object affects the atomic state, as well as how much the change in the quantum system impacts the motion. We will describe this coupling more quantitatively in Section 4 using the $\mathrm{NV}^{-}$center. 


\subsection{The $N V^{-}$Center}

The $\mathrm{NV}^{-}$color centers in diamond are of particular interest for spin-mechanics. First, thanks to the intense research activities around these color centers, triggered by their potentials in quantum information processing and the development of innovative sensors, their internal electronic and nuclear spin properties are very well understood. For the same reason, the control of diamond materials has progressed significantly over the past decade. Lastly, the electronic spin of the $\mathrm{NV}^{-}$center can easily be coupled with the diamond host matrix's motion using a magnetic field [14,19,46,56-59].

The physics and applications of the $\mathrm{NV}^{-}$centers have already been discussed in recent reviews $[60,61]$. We simply recall here the basics of $\mathrm{NV}^{-}$physics required for understanding the coupling to trapped particles.

The NV color center is a point defect inside the diamond matrix consisting of a substitutional nitrogen atom $(\mathrm{N})$ combined with a vacancy $(\mathrm{V})$ in one of the nearest neighboring sites of the diamond crystal lattice as depicted in Figure 2a. This defect behaves as an artificial atom hosted by the diamond matrix. It combines unique luminescence and spin properties. Indeed, because its energy levels are well within the large band gap of diamond, the NV center has an extremely stable luminescence in the near-infrared. It can be found in two different charge states: the $\mathrm{NV}^{-}$and $\mathrm{NV}^{0}$. The $\mathrm{NV}^{-}$zero-phonon line is at around $\lambda_{\mathrm{ZPL}}=637 \mathrm{~nm}$. It is associated with broad phonon sidebands that extend up to $\approx 750 \mathrm{~nm}$. The $\mathrm{NV}^{-}$is the most interesting for spin-mechanical interactions as we will see.

The $\mathrm{NV}^{-}$luminescence can readily be accessed using standard confocal microscopy, and can straightforwardly be adapted for levitation platforms. The photoluminescence from $\mathrm{NV}^{-}$centers in trapped diamonds under atmospheric conditions or in vacuum was observed by several groups $[53,54,62-65]$. Figure $2 \mathrm{~b}$ shows the photoluminescence from $\mathrm{NV}^{-}$centers in optical tweezers observed in [65]. Different pulsing sequences were used in order to mitigate PL quenching from the $1064 \mathrm{~nm}$ trapping laser. Note that, alternatively, photoluminescence (PL) quenching can be greatly reduced using other trapping laser wavelengths [55]. Further studies demonstrated trapping of nanodiamonds containing single $\mathrm{NV}^{-}$centers $[34,53,63]$. Figure $2 \mathrm{c}$ shows the autocorrelation function of the PL from a single $\mathrm{NV}^{-}$center inside a nanodiamond trapped in a Paul trap [34]. The antibunching dip at zero delay, below 0.5 is a proof for the presence of a single $\mathrm{NV}^{-}$center inside the trapped diamond, which opens a path towards non-gaussian spin-mechanics using the $\mathrm{NV}^{-}$spin.

a)

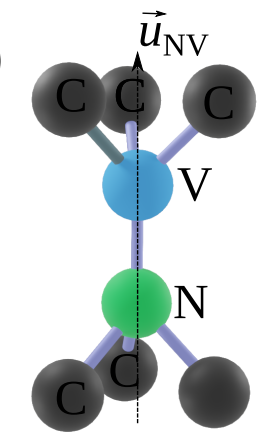

b)

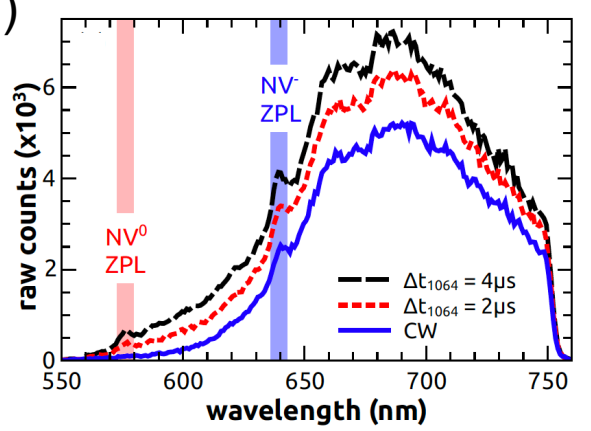

c)

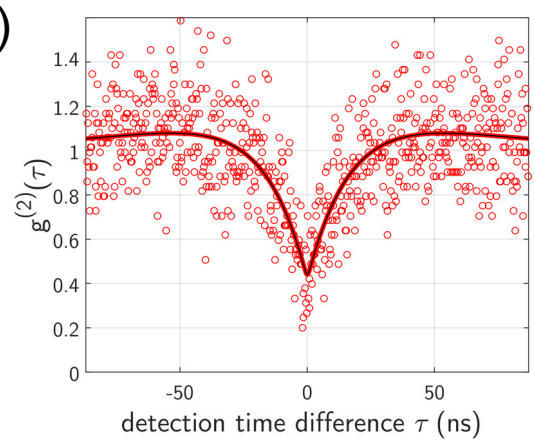

Figure 2. (a) Schematic of the crystalline structure of NV defect in diamond. The axis $N-V$ is the quantization axis. (b) Photoluminescence from $\mathrm{NV}^{-}$centers in an optical tweezer using three different excitation conditions. Adapted with permission from [65]. @ The Optical Society. (c) Autocorrelation function of photon emission from a single $\mathrm{NV}^{-}$center in a nanodiamond levitated in a Paul trap. Adapted with permission from [34]. Copyright 2020 American Chemical Society.

\subsection{The $N V^{-}$Center Electronic Spin}

In addition to the possibility of observing stable PL at ambient conditions, the $\mathrm{NV}^{-}$ center also carries a spin that can be manipulated at room temperature. The electronic spin 
of the $\mathrm{NV}^{-}$center in the ground state is a spin triplet $S=1$, with a quantization axis $\mathbf{u}_{\mathrm{NV}}$ enforced by the crystalline field around the defect. The spin projections along this axis are labelled with the quantum number $m_{s}$. Due to a spin-spin interaction between the two electrons in the ground state the $\left|m_{s}= \pm 1\right\rangle$ states are split from the $\left|m_{s}=0\right\rangle$ by the zero-field splitting $D \approx 2.88 \mathrm{GHz}$ (see Figure $3 \mathrm{a}$ ). These states are purely magnetic states, implying a very long longitudinal decay time $T_{1}$, on the order of milliseconds in typical diamond samples [66]. Note that in strained diamond, or in the presence of local electric fields [67], the states $\left|m_{s}= \pm 1\right\rangle$ can also be split, by a parameter often denoted $2 E$. This splitting is typically a few $\mathrm{MHz}$ in nanodiamonds. In practice, a magnetic field bias larger than $E$ is employed in order to reach large spin-mechanical couplings, so we neglect this zero-field splitting in the following.
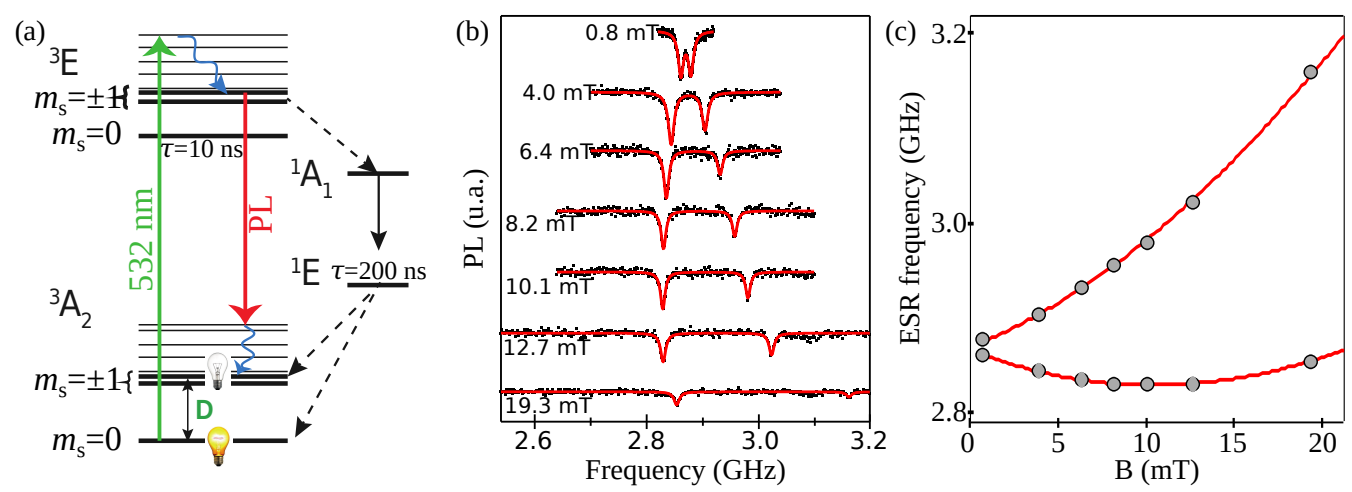

Figure 3. $\mathrm{NV}^{-}$center level structure. (a) Simplified electronic structure of the $\mathrm{NV}^{-}$center. (b) Example of optically detected magnetic resonance on a single $\mathrm{NV}^{-}$center in a bulk diamond. The magnetic field is applied with an angle $\theta=74^{\circ}$ with respect to the NV axis. (c) ESR frequencies from (b) (gray dots) compared with the full theory (red lines) obtained by computing the NV Hamiltonian eigenvalues (Equation (3)). Figure (b,c) are adapted from [68].

One interesting aspect of the $\mathrm{NV}^{-}$center resides in the possibility to optically initialize and read-out its electronic spin state. Indeed, under green laser irradiation the $\mathrm{NV}^{-}$ center is polarized in the $\left|m_{s}=0\right\rangle$ state, while the photoluminescence level depends on the populated spin state. The $\left|m_{s}=0\right\rangle$ state can be up to $30 \%$ brighter than the $\left|m_{s}= \pm 1\right\rangle$ states [61]. When a microwave tone is resonant with one of the transitions from $\left|m_{s}=0\right\rangle$ to $\left|m_{s}= \pm 1\right\rangle$, a drop of luminescence can then observed. This forms the basis of Optically Detected Magnetic Resonance (ODMR). The energy of these transitions can be found from the Hamiltonian of the electronic spin of the $\mathrm{NV}^{-}$center in the ground state, which reads

$$
\hat{\mathcal{H}} / \hbar=D \hat{S}_{z}^{2}+\gamma_{e} \hat{\mathbf{S}} \cdot \mathbf{B},
$$

where $\gamma_{e}$ is the gyromagnetic factor of the electron. Because of the zero-field splitting $D$ and the spin 1 character of the $\mathrm{NV}^{-}$center, the eigenstates of the Hamiltonian depend upon the angle between the magnetic field and the $\mathrm{NV}^{-}$center when $\gamma_{e} B<D$.

Typical single $\mathrm{NV}^{-}$ODMR spectra with fixed diamonds are presented in Figure $3 b$ showing the transitions from the $|0\rangle$ to $| \pm 1\rangle$ states as a drop of the PL. For this experiment, the $\mathrm{NV}^{-}$is at an angle of $74^{\circ}$ with respect to the magnetic field. Figure $3 \mathrm{c}$ shows the change in the frequency of the two transitions as a fonction of magnetic field as well as a numerical calculation using Equation (3).

The width of the observed electron spin resonance (ESR) dip is here limited by the dipolar coupling between the spin of $\mathrm{NV}^{-}$centers to other paramagnetic impurities in the diamond. The most dominant impurity is the substitutional nitrogen (P1 centers), with concentrations ranging from 100 to 500 ppm in high-pressure-high-temperature grown diamonds (see Section 8.1). Dipolar coupling between the $\mathrm{NV}^{-}$center and P1 centers typically lead to an inhomogeneous dephasing time $\Gamma_{2}^{*}$ on the order of 5 to $10 \mathrm{MHz}$ [69]. Ultimately, the linewidth of the ESR is of crucial importance, since it limits the measurement 
sensitivity as well as impacting the spin coupling strength to the mechanics. Nevertheless, as discussed in the Section 7, diamond growth allows fine control over impurities.

The frequency shifts of the spin resonances with magnetic field and their dependence with the magnetic field angle are important ingredients for coupling the center of mass and the angle of levitated particles to the $\mathrm{NV}^{-}$spin states, as we now discuss.

\section{Hamiltonian of the Spin-Mechanical System}

In the following, we consider a diamond containing a single $\mathrm{NV}^{-}$center in the presence of a homogeneous magnetic field as well as in a magnetic field gradient. We will consider the case of an $\mathrm{NV}^{-}$center in a trapped diamond but this analysis can be carried over to studies where an $\mathrm{NV}^{-}$center is coupled with a distant trapped magnet [35,70].

When the genuine quantum nature of single spins is not of interest, the following treatment can also straightforwardly be adapted to $N$ spins. If correlations between the NV spins inside the diamond from direct dipolar interactions [71-74], or resonator mediated interactions $[19,20,75]$ are neglected, the coupling strength can simply be multiplied by the number of spins. To be able to directly carry a single-spin analysis over to the ensemble case, one must also assume that the NV centers all have the same orientation in the diamond. In practice this is not the case since the $\mathrm{NV}^{-}$centers will typically be found with the same probability along the four [111] diamond directions. However, in the presence of a magnetic field that does not broaden the ESR width, the microwave can select only one of the eight resulting ESR transitions so that one can treat this problem using $N$ effectively spin $1 / 2$ systems in a single orientation with ESR frequencies that are within the inhomogeneous broadening $\Gamma_{2}^{*}$.

We will study the coupling between the spin and the two main degrees of freedom of a levitating mechanical oscillator: the center of mass motion (CoM) and the libration. We will assume that there is no coupling between these two degrees of freedom so that we can treat them separately. Note that this may not be true generally. For instance, in Paul traps, if the charge distribution has a non-zero dipole component, the center of mass and the angle may become coupled [76]. Similarly, shape anisotropy or birefringence of optically trapped particles can also induce a mode coupling [77,78]. These mechanisms are neglected here, but can be of interest for transferring the quantum state of one spin-coupled mode to another one.

\subsection{Coupling to the Center of Mass}

The center of mass motion (CoM) of the trapped particle can be coupled with the $\mathrm{NV}^{-}$spin using a magnetic field gradient [14]. Let us reduce the study of the spin-CoM coupling to a $1 \mathrm{D}$ problem. We assume that the NV axis is along the magnetic field. This greatly simplifies the problem by leaving aside mixing between the NV eigenstates and coupling between the CoM and the libration. When the magnetic field gradient is in the $z$ direction: $\mathbf{B}=B_{0} \mathbf{e}_{z}+\frac{\partial B_{z}}{\partial z} z \mathbf{e}_{z}$ to first order in the position. Under those assumptions, the Hamiltonian of the spin-mechanical system reads $\hat{\mathcal{H}}_{\text {com }}=\hat{\mathcal{H}}_{\text {mecha }}+\hat{\mathcal{H}}_{\mathrm{NV}}$, where $\hat{\mathcal{H}}_{\text {mecha }}=\frac{p_{z}^{2}}{2 m}+\frac{1}{2} m \omega_{z}^{2} z^{2}$ and $\hat{\mathcal{H}}_{\mathrm{NV}}=\hbar D \hat{S}_{z}^{2}+\hbar \gamma_{e}\left(B_{0}+\frac{\partial B_{z}}{\partial z} z\right) \hat{S}_{z}$.

Due to the Zeeman term in $\hat{\mathcal{H}}_{\mathrm{NV}}$, the magnetic energy is linearly proportional to the particle position. A magnetic force directly related to the magnetic field gradient and the chosen spin polarisation can thus be applied to the particle (see Section 6).

In the presence of an oscillating magnetic field $B_{\mu W}$ that is linearly polarized along the $x$ direction, the spin part of the Hamiltonian reads

$$
\hat{\mathcal{H}}_{\mathrm{NV}+\mu \mathrm{w}}=\hbar D \hat{S}_{z}^{2}+\hbar \gamma_{e}\left(B_{0}+\frac{\partial B_{z}}{\partial z} z\right) \hat{S}_{z}+\hbar \Omega \cos (\omega t) \hat{S}_{x}
$$


where $\Omega=\gamma_{e} B_{\mu W}$. Moving to the rotating frame at the microwave frequency $\omega$ through the unitary transform $\hat{U}=e^{i \omega t \hat{S}_{z}^{2}}$ and making a rotating wave approximation, we get

$$
\hat{\mathcal{H}}_{\mathrm{NV}+\mu \mathrm{w}}^{\prime}=\hbar(D-\omega) \hat{S}_{z}^{2}+\hbar \gamma_{e}\left(B_{0}+\frac{\partial B_{z}}{\partial z} z\right) \hat{S}_{z}+\hbar \frac{\Omega}{2} \hat{S}_{x}
$$

If $\gamma_{e} B_{0}$ is greater than both $2 E$ and $\Gamma_{2}^{*}$ and if the optical pumping process is stronger than the relaxation rate $T_{1}$ (see Section 3), resonantly tuning the microwave frequency to the $|0\rangle \leftrightarrow|+1\rangle(\operatorname{res} p .|-1\rangle)$ transition enables the $|-1\rangle$ (resp. $|+1\rangle$ ) state to be safely neglected. Choosing a microwave signal tuned to the transition $|0\rangle \leftrightarrow|+1\rangle$, we finally obtain the Hamiltonian of a two-level system coupled with a mechanical oscillator:

$$
\hat{\mathcal{H}}_{\text {total }}=\hat{\mathcal{H}}_{\text {mecha }}+\hat{\mathcal{H}}_{\text {Spin }}+\hat{\mathcal{H}}_{\text {Spin-mecha }}
$$

where $\hat{\mathcal{H}}_{\text {Spin }}=-\hbar \Delta|1\rangle\langle 1|+\hbar \frac{\Omega}{2}(|0\rangle\langle 1|+| 1\rangle\langle 0|)$ with the microwave detuning $\Delta=\omega-$ $D-\gamma_{e} B_{0}$ and $\hat{\mathcal{H}}_{\text {Spin-mecha }}=\hbar G_{z} z|1\rangle\langle 1|$ with the coupling constant $G_{z}=\gamma_{e} \frac{\partial B_{z}}{\partial z}$. Note that the latter has been redefined a $\Omega \equiv \frac{\Omega}{\sqrt{2}}$ to ensure normalisation of the $\hat{S}_{x}$ operator.

Before studying this Hamiltonian and the related experiments in more details, we will discuss the spin-coupling to the librational degree of freedom.

\subsection{Coupling to the Libration}

The theory behind spin-coupling to the libration has been presented in several papers $[19,46,57]$. Here, we derive a simplified Hamiltonian in the $\gamma_{e} B \ll D$ limit. Let us use the vectors $\left(0, \mathbf{e}_{x}, \mathbf{e}_{y}, \mathbf{e}_{z}\right)$ to specify the orientation of the laboratory frame and the vectors $\left(0, \mathbf{e}_{x^{\prime}}, \mathbf{e}_{y^{\prime}}, \mathbf{e}_{z^{\prime}}\right)$ to specify the particle frame. We choose $\mathbf{e}_{z}^{\prime}$ as the anisotropy axis of the $\mathrm{NV}^{-}$center in the crystalline structure of the diamond. The three Euler angles operators $(\hat{\phi}, \hat{\theta}, \hat{\psi})$ describing the angular position of the diamond are chosen in the $\left(z y^{\prime} z^{\prime \prime}\right)$ convention. The magnetic field is supposed to be homogeneous and its direction is fixed in the laboratory frame. It is chosen along the $z$ direction, so that $\mathbf{B}=B \mathbf{e}_{z}$.

The Hamiltonian of the spin-mechanical system in the laboratory frame reads

$$
\hat{\mathcal{H}}_{\text {lib }}=\frac{\hat{\mathbf{L}}^{2}}{2 I}+U(\hat{\phi}, \hat{\theta}, \hat{\psi})+\hbar D \hat{S}_{z^{\prime}}^{2}+\hbar \gamma_{e} B \hat{S}_{z},
$$

where $\hat{\mathbf{L}}$ is the angular momentum operator of the diamond in the laboratory frame, $U(\hat{\phi}, \hat{\theta}, \hat{\psi})$ is the angular confining potential and $\hat{S}_{z}, \hat{S}_{z^{\prime}}$ are $\mathrm{NV}^{-}$center spin operators. Contrary to when studying the coupling of the NV center to the center of mass, the NV direction is not necessarily fixed in the laboratory frame. This implies that the $\hat{S}_{z^{\prime}}$ operator depends on angular operators $(\hat{\phi}, \hat{\theta}, \hat{\psi})$ which do not commute with the diamond angular momentum operator $\hat{\mathbf{L}}$. Consequently, the commutator $\left[\hat{\mathbf{L}}^{2}, \hat{S}_{z^{\prime}}^{2}\right] \neq 0$ [79].

In the following, we will restrict the study to one librational mode that is assumed to be in the $\left(z z^{\prime}\right)$ plane formed by the magnetic field and spin direction. The diamond angular motion is parametrized by the nutation angle operator $\hat{\theta} . \theta^{\prime}$ represents the equilibrium angular position of the diamond.

The Hamiltonian of the simplified system reads

$$
\hat{\mathcal{H}}_{\text {lib }}=\frac{\hat{p}_{\theta}^{2}}{2 I}+\frac{1}{2} I \omega_{\theta}^{2}\left(\hat{\theta}-\theta^{\prime}\right)^{2}+\hbar D \hat{S}_{z^{\prime}}^{2}+\hbar \gamma_{e} B \hat{S}_{z}
$$

where $\hat{p}_{\theta}$ is the angular momentum operator along the $y$ axis. Moving to the particle frame through the unitary transformation $\hat{U}=e^{i \hat{\theta} \hat{S}_{y}}$ changes the Hamiltonian to

$$
\hat{\mathcal{H}}_{\text {lib }}^{\prime}=\frac{\left(\hat{p}_{\theta}-\hbar \hat{S}_{y}\right)^{2}}{2 I}+\frac{1}{2} I \omega_{\theta}^{2}\left(\hat{\theta}-\theta^{\prime}\right)^{2}+\hbar D \hat{S}_{z}^{2}+\hbar \gamma_{e} B\left(\cos \hat{\theta} \hat{S}_{z}-\sin \hat{\theta} \hat{S}_{x}\right) .
$$


In this frame, $\hat{p}_{\theta}$ is changed to $\hat{p}_{\theta}-\hbar \hat{S}_{y}$, which is the total angular momentum of the spin-mechanical system along the $y$ axis. It is the sum of the particle and the NV-spin angular momentum. This modified angular momentum could cause precession of the particle. Such a precession is predicted to be observable in levitated hard ferromagnetic nano-particles [80,81], with important applications in gyroscopy. For nano- or microparticles containing a smaller amount of spins, the total angular momenta correction $\hbar N \hat{S}_{y}$ is negligible. In order to estimate the relevance of this term in current experiments, one can note that $\left\langle\hat{p}_{\theta}\right\rangle \approx \sqrt{k T I}$ is far above $\hbar N$ even when using a highly-doped $(\approx 10 \mathrm{ppm})$ micron-sized diamond at temperatures above $\mu \mathrm{K}$. This condition will thus be verified for all smaller particles, since the maximal density of $\mathrm{NV}$ centres scales with the particle volume. In this considered temperature regime, we can thus treat the angle as a scalar and neglect the contribution from the angular spin momentum, bearing in mind that this approximation drops when using an ultra-cold oscillator.

The remaining step is to diagonalize the spin part of the Hamiltonian. Apart from in ref. [82], experiments currently operate in the regime $\gamma_{e} B \ll D$. We thus assume this condition to be fulfilled in the following. We also assume small mixing between the two $\mathrm{NV}^{-}$excited states, which is satisfied under the condition $\sin ^{2} \theta^{\prime} \ll \cos \theta^{\prime}$. Lastly, we change variable and shift the angle $\theta$ to the equilibrium position $\theta^{\prime}$. Under those assumptions, the Hamiltonian reads (see Appendix A or the detailed calculation):

$$
\hat{\mathcal{H}}_{\mathrm{lib}}^{\prime \prime \prime} \simeq \frac{p_{\theta}^{2}}{2 I}+\frac{1}{2} I \omega_{\theta}^{2} \theta^{2}+\hbar\left(\omega_{+1}(\theta)\left|+1^{\prime}\right\rangle\left\langle+1^{\prime}\left|+\omega_{0}(\theta)\right| 0^{\prime}\right\rangle\left\langle 0^{\prime}\left|+\omega_{-1}(\theta)\right|-1^{\prime}\right\rangle\left\langle-1^{\prime}\right|\right) .
$$

The expression of the new eigenstates $\left| \pm 1^{\prime}\right\rangle$ and $\left|0^{\prime}\right\rangle$ is listed in Appendix A. The frequencies $\omega_{i}(\theta)=\omega_{i}+\beta_{i} \theta$ of the spin resonance are plotted as a function of the angle $\theta$ for two different magnetic field values in Figure 4a.

(a)

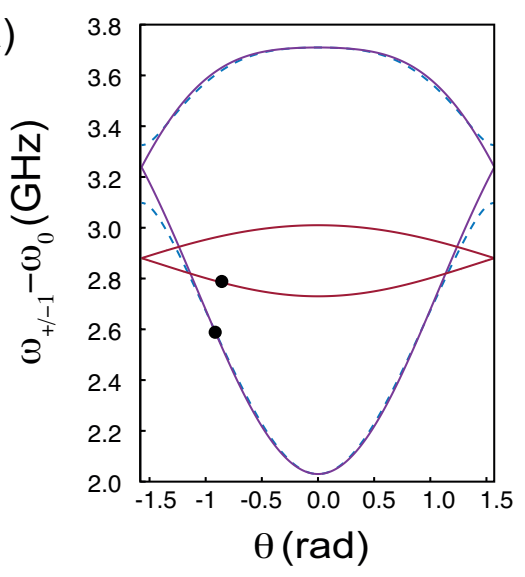

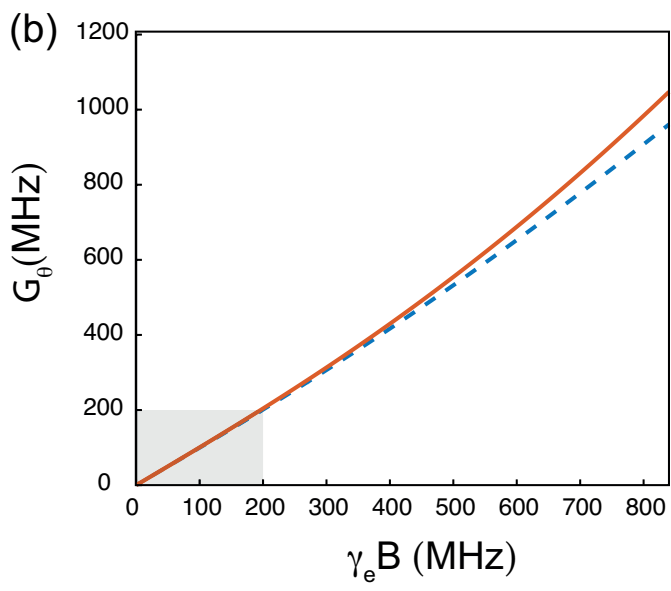

Figure 4. (a) Electronic spin transition energies of the $\mathrm{NV}^{-}$center as a function of the angle $\theta$ for magnetic fields of $50 \mathrm{G}$ (brown lines) and $300 \mathrm{G}$ (blue lines). The black dots indicate the optimum angles for the largest spin-torques. (b) Optimum coupling strength $G_{\theta}$ on the $|0\rangle$ to $|-1\rangle$ transition as a function of the external magnetic field. The shaded area corresponds to the magnetic field values for which the optimized coupling strength is very close to $\gamma_{e} B$. In both plots, numerical calculations without approximations are shown by dashed lines.

It was shown in [68] that the pumping process is not modified to first order in the transverse magnetic field. We can thus neglect the mixing contribution from the optical pumping process of the $\mathrm{NV}^{-}$center. This is also manifest in Figure $3 \mathrm{~b}$ where the contrast of the ODMR drops significantly only with magnetic field values above $\approx 10 \mathrm{mT}$. 
Similar to the calculation for the center of mass (CoM), we obtain the Hamiltonian (see Appendix A):

$$
\hat{\mathcal{H}}_{\mathrm{NV}+\mu \mathrm{w}}^{\prime} / \hbar \simeq-\Delta_{+1}(\theta)\left|+1^{\prime}\right\rangle\left\langle+1^{\prime}\left|-\Delta_{0}(\theta)\right| 0^{\prime}\right\rangle\left\langle 0^{\prime}\left|-\Delta_{-1}(\theta)\right|-1^{\prime}\right\rangle\left\langle-1^{\prime}\right|+\frac{\Omega}{2} \hat{S}_{x},
$$

where $\Delta_{+1}(\theta)=\omega-\omega_{+1}(\theta), \Delta_{-1}(\theta)=\omega-\omega_{-1}(\theta)$ and $\Delta_{0}(\theta)=-\omega_{0}(\theta)$. We redefined $\Omega \equiv \Omega \cos \theta^{\prime}$. The eigenstates are listed in Appendix A. Choosing a microwave tuned close to resonance with the $\left|0^{\prime}\right\rangle$ to $\left|+1^{\prime}\right\rangle$ transition allows us to restrict the study to the Hamiltonian of a two-level system

$$
\hat{\mathcal{H}}_{\mathrm{NV}+\mu \mathrm{w}}^{\prime \prime} / \hbar \simeq\left(-\Delta+\beta_{1} \theta\right)\left|1^{\prime}\right\rangle\left\langle 1^{\prime}\left|+\beta_{0} \theta\right| 0^{\prime}\right\rangle\left\langle 0^{\prime}\right|+\frac{\Omega}{2}\left(|0\rangle\left\langle 1^{\prime}|+| 1^{\prime}\right\rangle\langle 0|\right) .
$$

Here $\Delta=\Delta_{+1}-\Delta_{0}$ and $\Omega \equiv \frac{\Omega}{\sqrt{2}}$ to take into account the $\sqrt{2}$ factor in the $\hat{S}_{x}$ operator of the spin-1 system. We have fixed the energy reference to be the energy of the $\left|0^{\prime}\right\rangle$ state.

The second term $\beta_{0} \theta\left|0^{\prime}\right\rangle\left\langle 0^{\prime}\right|$ operates as a small shift of the angular position when the $\mathrm{NV}^{-}$ground state is populated and can be interpreted as a consequence of van Vleck paramagnetism. The mixing between the ground and excited states induced by the transverse magnetic field indeed generates a non-zero magnetic moment in the $\mathrm{NV}^{-}$center $[82,83]$. This term gives rise to a new equilibrium position in experiments because the $\left|0^{\prime}\right\rangle$ is populated by the green laser.

At this point, a word of caution is in order if one wishes to translate the above description to ensembles of NV centers. We indeed need to check for the consistency of our initial assumptions about independent NV directions in the presence of strong van Vleck paramagnetism. This paramagnetism is present in the absence of microwave excitation, so all NV orientations contribute to give a spin-torque in the ground state, the extent of which depends upon the transverse magnetic field amplitude for each orientation. When including the four orientations, the first-order effect is a slight reduction of the van Vleck paramagnetic susceptibility that can be estimated for a single orientation. This is due to an overall spatial averaging of the magnetizations from the four $\mathrm{NV}$ orientations, which should be recast in $\beta_{0}$. The reader can find more information in [83].

Let us redefine the center of the mechanical resonator to be around the equilibrium angular position when the population is in the $\left|0^{\prime}\right\rangle$ state by including the van Vleck shift in $\Delta$. Then, by defining $G_{\theta}=\beta_{1}-\beta_{0}$ to be the single-spin mechanical constant, the Hamiltonian of the system can finally be written as the simple two-level atom Hamiltonian:

$$
\hat{\mathcal{H}}_{\mathrm{NV}+\mu \mathrm{w}}^{\prime \prime} / \hbar=\left(\begin{array}{cc}
-\Delta(\theta) & \Omega / 2 \\
\Omega / 2 & 0
\end{array}\right)
$$

where $\Delta(\theta)=\Delta-G_{\theta} \theta$ is the frequency difference between the states $\left|0^{\prime}\right\rangle$ and $\left|1^{\prime}\right\rangle$. The Hamiltonian for the librational mode is thus the same as for the center of mass (Equation (6)), when replacing the coupling constant $G_{z}$ by $G_{\theta}$.

The optimum coupling strength $G_{\theta}$ on the $|0\rangle$ to $|-1\rangle$ transition is plotted in Figure $4 \mathrm{~b}$. Ones finds that $G_{\theta} \approx \gamma_{e} B$ when $\gamma_{e} B<200 \mathrm{MHz}$. There is a good match between analytical numerical calculations in this range of magnetic fields. Slight deviations between analytical treatment and numerical calculations are visible when $\gamma_{e} B>200 \mathrm{MHz}$ due to stronger state mixing as $\gamma_{e} B$ approaches $D$.

\section{Sensing the Motion of a Trapped Particle Using $\mathbf{N V}^{-}$Centers}

As we just described, the ESR frequencies depend strongly on the angle of the homogeneous magnetic field with respect to the NV axis, and on the center of mass in a magnetic field gradient. In the presence of a microwave drive on the diamond, the change in the photoluminescence from the $\mathrm{NV}^{-}$centers can then be used as a marker of the center of mass motion of diamonds or distant ferromagnetic particles, as pioneered in experiments with tethered oscillators $[16,17]$. With trapped diamond particles, the angular dependence 
of the NV center energy levels also means that it is possible to detect their full rotation and even their librational motion.

Figure 5a shows an ODMR obtained from optically trapped diamonds in water featuring broad lines due to significant angular Brownian motion in the presence of a magnetic field [84]. Similar ODMR shapes were observed in [54,63]. These results show that NV centers are an efficient tool to measure the rotation of particles. Further, the fully rotating regime may also shed new light on geometric phases acquired by NV centers as well as offering perspectives for efficient sensing of magnetic fields in the rotating frame [85].

(a)

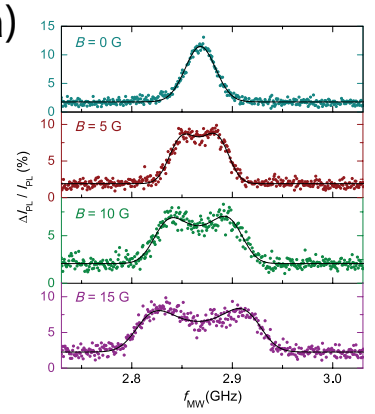

(b)

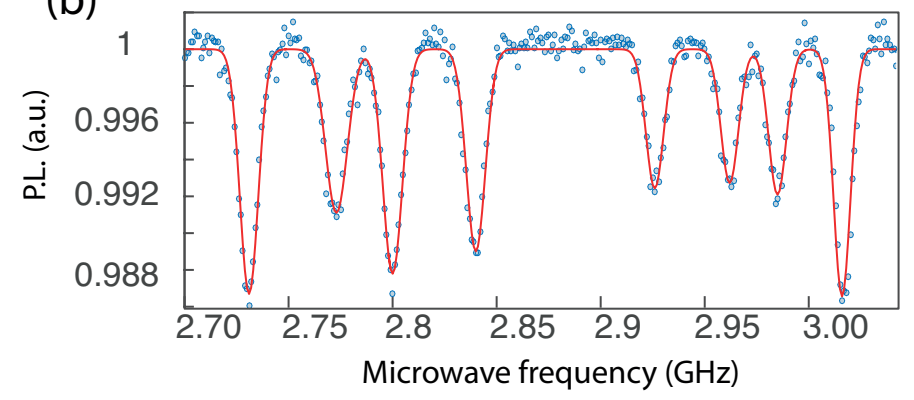

Figure 5. (a) ODMR for small ensembles of nano-diamond trapped in water, under increasing magnetic fields. Adapted form [84]. (b) ODMR spectrum for a small ensemble of $\mathrm{NV}^{-}$centers in a levitated micro-diamond in a magnetic field of about $50 \mathrm{G}$. Eight peaks corresponding to the four orientations of the $\mathrm{NV}^{-}$centers, are observed. Reprinted figure with permission from [86]. Copyright 2018 by the American Physical Society.

Note that, additionally, control of the trapping laser polarisation allows tuning the NV angles [87]. In [54,86], ODMR from diamond particles in Paul traps was also reported on angularly stable micro-diamonds (with typical librational frequencies in the $\mathrm{kHz}$ range). Figure $5 \mathrm{~b}$ shows the $\mathrm{PL}$ of $\mathrm{NV}^{-}$centers from a diamond containing about $1000 \mathrm{NV}^{-}$centers. Eight ODMR lines were observed due to the four projections of the magnetic field onto the four possible NV axes in the diamond cristalline structure and thanks to the efficient particle angular confinement. Assuming thermalization with a gas at a temperature close to $\mathrm{T}=300 \mathrm{~K}$, the angular standard deviation is $\Delta \theta \approx 1 \mathrm{mrad}$ (see Section 2 ). The expected extra broadening of the ODMR lines in a magnetic field $B \approx 50 \mathrm{G}$ is on the order of $G_{\theta} \Delta \theta \approx \gamma B \Delta \theta=150 \mathrm{kHz}$. This value is smaller than the width given by the dipolar coupling between the NV and P1 centers (see Section 3).

Two recent experiments have even shown read out of the harmonic motion of a trapped particle using NV centers [35,70]. In the first experiment [70], a hybrid diamond-nickel particle was levitated in a Paul trap. The $\mathrm{NV}^{-}$centers in the diamond were employed to read-out the librational motion. In the second experiment [35], a magnet was levitating on top of superconducting sheet and a diamond containing a single $\mathrm{NV}^{-}$center was brought in the vicinity of the magnet to read-out its center of mass motion.

In order for NV centers to efficiently detect the center of mass motion, Gieseler et al. [35] used broadband magnetic noise to excite the motion of the trapped micro-magnet (diameter $\approx 15 \mu \mathrm{m}$ ). The magnetic field thermal noise at the NV location, generated by the $100 \mu \mathrm{m}$ distant driven trapped magnet, was then observed in the power spectral density (PSD) of the NV PL evolution. Thanks to the high quality factor of the oscillator $\left(Q \approx 10^{6}\right)$, tuning a microwave to the blue side of the ODMR signal, as shown in the top of Figure 6a, resulted in a sharp peak at the mechanical oscillator frequency.

Similarly, Huillery et al. [70] reported NV-based detection of the motion of a $10 \mu \mathrm{m}$ hybrid ferromagnet/diamond particle. The latter was levitated in a Paul trap and the libration was detected in the time domain after parametric excitation of the magnetically confined librational mode. Figure $6 \mathrm{~b}$ shows the ODMR (top trace) and the ring down of the librational mode (below) detected both using scattered light and the NV PL. Notably, 
the PL change was delayed with respect to the instantaneous particle motion due to a time lag between the motion and the magnetization.

These studies open a path towards the NV read-out of the Brownian motion of trapped harmonic oscillators. To reach this limit, more involved dynamical decoupling (DDC) techniques [17] could be used, with perspectives for attaining the zero-point motion sensitivity of the oscillator. Rabi oscillations, Ramsey and spin echoes from NV centers in a trapped diamond have in fact been observed already without significant deterioration of the $T_{2}$ from either the charge noise or the angular Brownian motion [86]. Rabi oscillations were also observed in [53] using an optically trapped nanodiamond under weak magnetic fields. DDC would, however, require to enter the regime where the trapping frequency of the trapped mechanical oscillator exceeds the decoherence rate $\Gamma_{2}^{*}$ of the NV spins. We discuss ways to achieve this in Section 8.1.

(a)
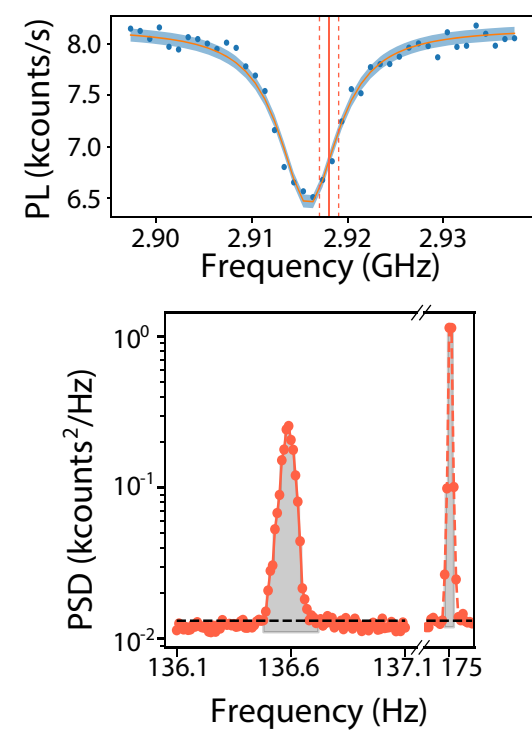

(b)
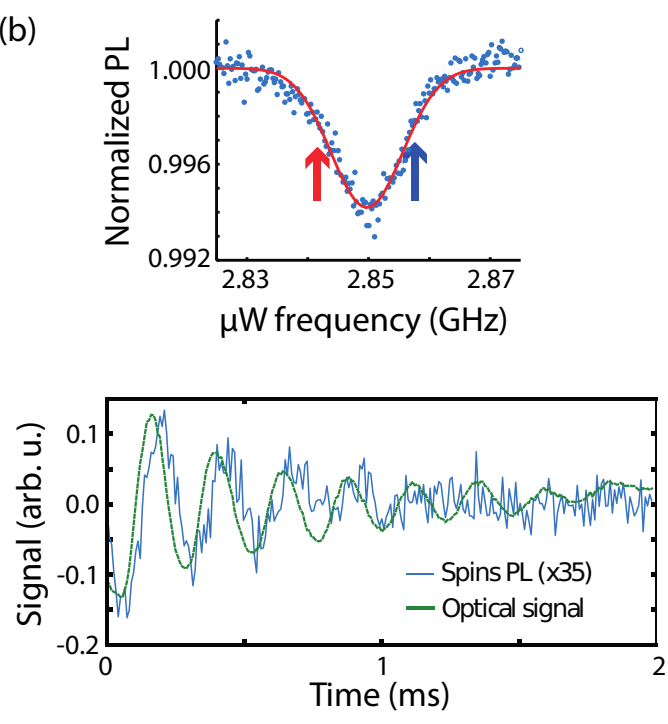

Figure 6. $\mathrm{NV}^{-}$sensing of the motion of trapped particles. (a) Top trace: ODMR on a $\left|m_{s}=0\right\rangle$ to $\left|m_{s}=+1\right\rangle$ transition from a fixed single $\mathrm{NV}^{-}$center, located $100 \mu \mathrm{m}$ away from the levitating magnet. Below: PSD of the NV fluorescence signal when a microwave is tuned to the blue side of the ODMR peak. The right narrow peak shows the magnet motion when the microwave is modulated at $175 \mathrm{~Hz}$. Adapted from [35]. (b) Top trace: ODMR on a $\left|m_{s}=0\right\rangle$ to $\left|m_{s}=-1\right\rangle$ transition from several $\mathrm{NV}^{-}$centers inside a hybrid ferro-diamond particle. Bottom trace: Time trace showing the librational ring-down from the hybrid structure. The detection was realized using both the laser scattered light and the PL. Reprinted figure with permission from [70]. Copyright 2020 by the American Physical Society.

\section{Magnetic Forces and Torques on a Trapped Particle from the Spin of $\mathrm{NV}^{-}$Centers}

We have discussed experiments where the trapped particle motion was read-out by $\mathrm{NV}$ spin states. In order to strongly couple a mechanical oscillator to spins, however, not only should the mechanical motion affect the spin state, but the spin should also alter the motion.

In this section, we review work on the reverse process where NV centers act on the motion. We start by a discussion on optimum sensing of the force and the torque induced by NV centers.

\subsection{Force and Torque Sensitivity}

Presently, direct optical read-out of the motion of trapped particles using scattered light is more efficient than using embedded NV centers. Most experiments that use optical read-out are currently in the regime where the influence of collisions with the background gas dominates. The detection noise is thus determined by the resulting Brownian motion of the levitating object. Note that the ultimate sensitivity should be given by the quantum 
back-action of the measurement under low vacuum. This limited was recently reached in experiments with trapped nano-spheres [88,89].

The most sensitive way to detect an external torque, with current technology, is to use optical interferometric detection and to modulate the torque at the mechanical frequency of the oscillator. Modulation of the amplitude of the NV induced spin-torque can be done straightforwardly by modulating the microwave tone that excites them. The minimum torque $\tau_{s}^{\mathrm{min}}$ that can be detected in a time $\delta T$ is then obtained by balancing the resulting signal amplitude and the standard deviation of the Brownian motion noise. One finds

$$
\tau_{s}^{\min } \sqrt{\delta T}=\sqrt{4 k T \gamma_{\theta} I}=\sqrt{\frac{4 k T K_{t}}{Q \omega_{\theta}}},
$$

where $Q=\omega_{\theta} / \gamma_{\theta}$ is the quality factor of the mechanical oscillator libration, and $K_{t}$ is the trap rigidity, namely $I \omega_{\theta}^{2}$.

Tethered torque sensors with sensitivities in the $10^{-23} \mathrm{~N} \cdot \mathrm{m} / \sqrt{\mathrm{Hz}}$ range are realized nowadays with state-of-the-art nano-fabricated oscillators [90]. The largest sensitivities with levitating systems have been achieved using levitating silica nanospheres that are attached to form a dumbbell [91]. The authors reached a record sensitivity of $10^{-28} \mathrm{~N} \cdot \mathrm{m} / \sqrt{\mathrm{Hz}}$. Trapped cristalline particles currently feature a lower torque sensitivity than trapped amorphous particles, partly because of their currently lower quality factor. A sensitivity of $10^{-23} \mathrm{~N} \cdot \mathrm{m} / \sqrt{\mathrm{Hz}}$ was attained in [70] using soft ferromagnetic particles at room temperature and at pressure levels in the $10^{-2}$ mbar range, very close to the state-of-the-art torque sensing obtained at dilution fridge temperatures [90].

A similar formula can be obtained for the smallest detectable force acting on the center of mass mode, by replacing $Q$ with $\omega_{z} / \gamma_{z}, K_{t}$ by $m \omega_{z}^{2}$ and $I$ by $m$. Sensitivities in the zeptonewton $/ \sqrt{\mathrm{Hz}}$ range were reported experimentally $[28,92,93]$. Very large sensitivities are also predicted for a magnet levitating on top of a punctured superconductor sheet in the Meissner state [94]. Amongst all spin-mechanical systems, trapped magnets currently feature record sensitivities in the $10^{-18} \mathrm{~N} / \sqrt{\mathrm{Hz}}$ range $[35,44]$. Note that at the low pressure levels employed in these experiments $\left(\approx 10^{-5}\right.$ mbar $)$, damping is not determined by collisions with the background gas so the above model may not apply directly.

These last achievements not only show the capabilities of levitating systems, close to the sensing capabilities of MEMS, but also offer immediate perspectives for entering the quantum regime by coupling magnets to distant $\mathrm{NV}$ centers.

\subsection{Observing NV Static Spin-Dependent Torque and Force}

In this section, we discuss the parameters required for observing spin-dependent forces and torques on a trapped particle. We focus on the expected static shifts, assuming that the $\mathrm{NV}^{-}$centers have a lifetime that is greater than the typical time required to shift the angle or the center of mass. The latter is typically on the order of the period of the potential.

\subsubsection{Angular Displacement Using $\mathrm{NV}^{-}$Centers}

Using Equation (10), one finds that the torque applied to the diamond in the magnetic state $\left|-1^{\prime}\right\rangle$ reads

$$
\tau_{s}=-\left\langle\frac{\partial \hat{\mathcal{H}}_{\mathrm{lib}}^{\prime \prime \prime}}{\partial \theta}\right\rangle=-\hbar N \beta_{-1} .
$$

The optimum shift will be found for $\theta=\pi / 2$, where $\tau_{s}=-\hbar N \gamma_{e} B$ so the largest single spin torque can be $\approx 10^{-25} \mathrm{~N} \cdot \mathrm{m}$.

Let us consider a particle with a diameter of $15 \mu \mathrm{m}$ undergoing Brownian motion in a harmonic trap under $0.1 \mathrm{mbar}$. Stable harmonic confinement is readily attainted at $300 \mathrm{~K}$ in a Paul trap with these parameters [45], enabling a sensitivity of $\approx 10^{-21} \mathrm{~N} \cdot \mathrm{m} / \sqrt{\mathrm{Hz}}$. This sensitivity is far from enabling single spin torque detection in a reasonable amount of time. 
Reference [45] reported the observation of spin-torque in this parameter regime, albeit using $10^{9}$ spins that were all identically coupled with the libration. Figure $7 \mathrm{~b}$ demonstrates mechanical detection of the $\approx 10^{-19} \mathrm{~N} \cdot \mathrm{m}$ spin torque averaged over a few minutes with a large signal to noise ratio (taken from [45]), revealing a novel efficient method to probe ESR from NV centers.

In order to approach the single spin torque level, particles with much smaller moment of inertia must be used. A levitating particle with a diameter of $100 \mathrm{~nm}$ and a modest $Q$ factor of $10^{4}$ would be sufficient to reach sensitivities in the $10^{-25} \mathrm{~N} \cdot \mathrm{m} / \sqrt{\mathrm{Hz}}$ range. Single-spin torque could then be discerned within one second in this experimentally achievable regime.
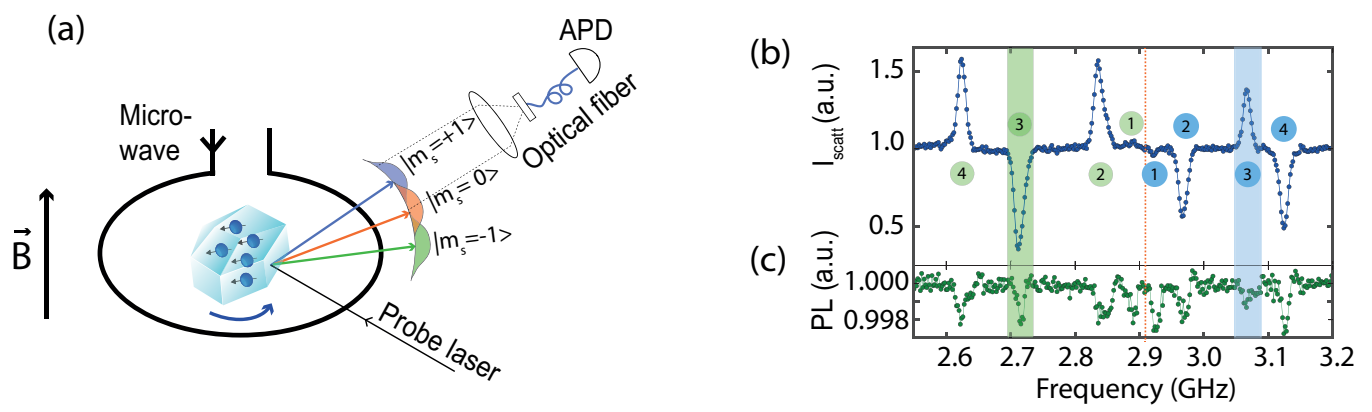

Figure 7. (a) Sketch showing a NV-doped diamond in a Paul trap where the probe laser reflection angle from the diamond surface depends on the spin state. (b) Mechanically detected magnetic resonance observed by scanning the microwave tone about the NV transitions. (c) ODMR in similar experimental conditions. Adapted from [45].

\subsubsection{Center of Mass Displacement Using NV ${ }^{-}$Centers}

A similar discussion can be made to estimate the magnitude of the magnetic force on the CoM of a trapped diamond subjected to a magnetic field gradient

$$
F_{S}=-\left\langle\frac{\partial \hat{\mathcal{H}}_{\mathrm{com}}}{\partial z}\right\rangle=-\hbar N \gamma_{e} \frac{\partial B_{z}}{\partial z} .
$$

Under a gradient of $10^{5} \mathrm{~T} / \mathrm{m}$, one finds a single-spin force of $10^{-19} \mathrm{~N}$. These large gradients typically require trapped micron-size magnets a few hundreds of nanometers from the NV center. Although less attractive for quantum applications, ensembles of spins could be used to bypass this technical difficulty.

The spin-dependent force has not been observed thus far using $\mathrm{NV}^{-}$centers to the best of our knowledge, even with large ensembles of spins. Even if strong magnetic field gradients can be achieved, it may induce a large inhomogeneous broadening when using ensembles, which will forbid efficient microwave excitation of the whole ensemble of $\mathrm{NV}^{-}$centers in the selected orientation. The magnetic field gradient will thus be bounded by the microwave excitation Fourier width $\delta v$ in a pulsed excitation scheme, so that $\partial B_{z} /\left.\partial z\right|_{\max }=\Delta v / \gamma_{e} d$, where $d$ is the diameter of the probed spin-ensemble. Note that $\Delta v$ can reach more than $100 \mathrm{MHz}$ using high power amplifiers and dedicated fast electronics [95].

One further difficulty with NV centers coupled with trapped particles is to distinguish the center of mass motion from spin-induced torques. Indeed, a magnetic field offset is required in order to magnetize the $\mathrm{NV}^{-}$centers with a microwave which will rotate the trapped particle. The spin-induced angular displacements may thus contribute at the same level as the center of mass shifts, which complicates measurement analyses. One solution is to align the magnetic field along the [111] direction of the diamond, where no torque should be applied to the particle. This is a notoriously difficult task when using levitating particles where the angle between the diamond crystalline axes and the main trap axes cannot not always be controlled. 
Let us conclude by adding that single-spin forces were measured using Magnetic Resonance Force Microscopy (MRFM) in [96]. Acquisition times of many hours and carefully engineered modulation techniques were employed. It is likely that significant progress can be made by applying these techniques to spin-mechanics with NV centers, with fascinating prospects for controlling the motion of micro-objects using single long-lived qubits.

\section{Dynamical Resonant Spin-Mechanical Interaction}

Spin-mechanical systems can show richer physics than just static torques and forces. When calculating the torque and force in Sections 5 and 6, it was implicitly assumed that the spin population does not change after applying the microwave excitation. This is not the case in the strong spin-mechanical coupling regime. Indeed, after the microwave excites the spin and gives rise to a torque (or to a force), the particle angle (or position) changes, which in turn changes the microwave detuning with respect to the ESR, hence the magnetization. We discuss the resulting bistable and spin-spring effects below.

Further, when the spin-torque resulting from the combined laser and microwave induced magnetization is lagging behind the motion, exchange of heat between the spin and the mechanical oscillator can take place. Experiments have recently entered this regime [45].

We provide a simplified analytical theory of such spin dynamical back-action. We focus on the angular degree of freedom, but the calculation can be straightforwardly carried out for the center of mass by making the replacement $\left(\theta, G_{\theta}\right) \rightarrow\left(z, G_{z}\right)$.

\subsection{Bistability and Spin-Spring Effect}

As in opto-mechanics, bistability and modified spring constants can occur. We first describe these two effects, in the limit where the microwave magnetization and the laser polarisation rates are faster than the mechanical oscillator frequency. This means that no energy exchange between the motion and the spin can take place.

\subsubsection{Spin-Bistability}

The torque $\tau_{s}$ exerted on the particle due to the spin-mechanical coupling can be evaluated using Equation (13). We obtain

$$
\hat{\tau}_{s}=-\frac{\partial \hat{\mathcal{H}}_{\mathrm{NV}+\mu \mathrm{w}}^{\prime \prime}}{\partial \theta}=-\hbar N G_{\theta}\left|1^{\prime}\right\rangle\left\langle 1^{\prime}\right| .
$$

In the dispersive limit where $\Omega \ll \Delta(\theta)$, the spins are mostly in the lowest energy eigenstate $|-\rangle$ of $\hat{\mathcal{H}}_{\mathrm{NV}+\mu \mathrm{w}}^{\prime \prime}$ where the torque reads:

$$
\tau_{s}=\left\langle-\left|\hat{\tau}_{s}\right|-\right\rangle=-\hbar N G_{\theta}\left|\left\langle-\mid 1^{\prime}\right\rangle\right|^{2} \approx-\hbar N G_{\theta}\left(\frac{\Omega}{\Delta(\theta)}\right)^{2} .
$$

This spin torque can be added to the restoring torque of the levitating system. The new angular stable position can be found by solving the equation $\tau_{s}+\tau_{\text {trapping }}=0$. It gives rise to a third degree polynomial equation for $\theta$

$$
\hbar N G_{\theta} \Omega^{2}+I \omega_{\theta}^{2} \theta\left(\Delta+G_{\theta} \theta\right)^{2}=0 .
$$

In the very same way as in opto-mechanics, two stable solutions for $\theta$ can be found when $\Delta<0$. Angular bistability can then occur when the microwave is swept across the ESR transition.

\subsubsection{Dynamical Backaction: Spin-Spring Effect}

Linearizing about an equilibrium position $\theta_{0}$, and introducing the detuning $\bar{\Delta}=\Delta-G_{\theta} \theta_{0}$, we get

$$
\left\langle-\left|\hat{\tau}_{s}\right|-\right\rangle \approx \tau_{s, 0}+K_{s}\left(\theta-\theta_{0}\right),
$$


where

$$
\tau_{s, 0}=\hbar N G_{\theta}\left(\frac{\Omega}{\bar{\Delta}}\right)^{2} \text { and } K_{s}=-2 \hbar N G_{\theta}^{2} \frac{\Omega^{2}}{\bar{\Delta}^{3}},
$$

in the limit of small angle shifts. This expression predicts a restoring torque in the limit where $\bar{\Delta}>0$ (blue detuned with respect to the spin transition).

In the presence of the green laser light, transitions from the dressed spin states will alter this predicted shift. We will estimate it together with the spin-cooling effect using a density matrix formulation in the following.

\subsection{Spin-Cooling}

In the above estimation, we assumed that the spins react immediately to a change in the motion. When the spin torque lags behind the motion, friction forces can alter the motional temperature and lead to spin-cooling, as calculated in [57] and observed in [45]. In order to evaluate the dynamical back-action from the spins to the mechanical oscillator with retardation, one will include the dissipation of the electronic spin. Lastly, since most experiments are operating in the so-called adiabatic limit where the frequency of the mechanical oscillator is much smaller than the spin dephasing rate, we will consider this regime for simplicity and discuss the corresponding limits to spin-cooling.

\subsubsection{Equations of Motion}

We will again assume that the microwave is tuned to the $\left|0^{\prime}\right\rangle$ to $\left|1^{\prime}\right\rangle$ transition. When the longitudinal decay time $T_{1} \approx \mathrm{ms}$ is longer than the time $1 / \gamma_{\text {las }} \ll 100 \mu$ s it takes for laser polarizing the NV spin, the magnetic state $\left|-1^{\prime}\right\rangle$ is not populated. This can be ensured quite straightforwardly experimentally, using laser powers in the hundreds of $\mu \mathrm{W}$ range with standard microscope objectives. We will assume that this is the case here. We can thus reduce the study to the two level system described in Equation (13).

The von Neumann equation for the reduced two-by-two spin density matrix $\hat{\rho}$ reads

$$
\begin{aligned}
& \frac{\partial \rho_{10}}{\partial t}=\left(-\Gamma_{2}^{*}+i \Delta(\theta)\right) \rho_{10}+i \frac{\Omega}{2}\left(2 \rho_{11}-1\right) \\
& \frac{\partial \rho_{11}}{\partial t}=-\gamma_{\text {las }} \rho_{11}+i \frac{\Omega}{2}\left(\rho_{10}-\rho_{10}^{*}\right),
\end{aligned}
$$

where $\Gamma_{2}^{*}$ is the inhomogeneous broadening of the $\mathrm{NV}^{-}$center and $\gamma_{\text {las }}$ is the optical pumping rate to the $\left|0^{\prime}\right\rangle$ state. We also assumed $\gamma_{\text {las }} / 2 \ll \Gamma_{2}^{*}$, which is largely satisfied in practice. Note that these equations are valid when the broadening is purely homogeneous and of a Markovian nature. In general, the $\mathrm{NV}^{-}$centers couple to slowly fluctuating spin baths that generally implies Gaussian ESR lineshapes.

The above equations are coupled with the equation of motion of the particle via

$$
I \frac{\partial^{2} \theta}{\partial t^{2}}+I \gamma \frac{\partial \theta}{\partial t}+I \omega_{\theta}^{2} \theta=\left\langle\hat{\tau}_{s}\right\rangle_{\mathcal{B}}+\tau_{L}
$$

where $\left\langle\hat{\tau}_{s}\right\rangle_{\mathcal{B}}=-\hbar N G_{\theta} \rho_{11}$ and $\mathcal{B}$ accounts for incoherent laser excitation to the ground state, as well as pure dephasing due to dipolar coupling to the P1 centers. Because of the $\theta$ dependency in $\Delta$, this system of equations is nonlinear. Here, we study the system dynamics around a steady-state, which will linearize the set of equations. 


\subsubsection{Stationary Solutions}

We introduce the steady-state quantities $\rho_{11}^{0}=\left\langle\rho_{11}\right\rangle, \rho_{10}^{0}=\left\langle\rho_{10}\right\rangle$, and $\theta_{0}=\langle\theta\rangle$, where 〈.) denotes time averaging. Writing the incoherent pumping rate to the magnetic state at the angle $\theta_{0}$ as $\Gamma_{0}=\frac{\Omega^{2} \Gamma_{2}^{*}}{\Gamma_{2}^{* 2}+\bar{\Delta}^{2}}$, we get:

$$
\rho_{11}^{0}=\frac{1}{2} \frac{\Gamma_{0}}{\gamma_{\text {las }}+\Gamma_{0}} .
$$

Using Equation (24), one finds the steady state solution for the angle to be

$$
I \omega_{\theta}^{2} \theta_{0}=\left\langle\hat{\tau}_{s}\right\rangle_{\mathcal{B}}^{0}=-\hbar N G_{\theta} \rho_{11}^{0} .
$$

This last equation gives a third degree polynomial equation for $\theta_{0}$. Depending on the microwave detuning, there can either be one or two stable solutions for $\theta_{0}$.

\subsubsection{Effective Susceptibility}

Writing each spin and angle parameters in Equations (22)-(24) as $f(t)=f^{0}+\delta f(t)$ and transforming them to the Fourier domain, these equations can be recast into the compact expression $\delta \theta(\omega)=\chi_{\text {eff }}(\omega) \delta \tau_{L}(\omega)$, where the susceptibility $\chi_{\text {eff }}(\omega)$ reads

$$
\chi_{\mathrm{eff}}(\omega)=\frac{1}{I\left(\omega_{\theta}^{2}-\omega^{2}-i \omega \gamma\right)-K_{s}(\omega)} .
$$

The quantity $K_{s}(\omega)$ is a dynamical spin-rigidity which quantifies the response of the particle angle to a change in the spin-torque. The real part of $K_{s}(\omega)$ gives rise to a shift of the mechanical oscillator frequency while the imaginary part gives rise to a damping of the mechanical motion. We can rewrite the susceptibility in the more condensed form

$$
\chi_{\mathrm{eff}}(\omega)=\frac{1}{I\left(\tilde{\omega}_{\theta}^{2}-\omega^{2}-i \omega \tilde{\gamma}\right)},
$$

with

$$
\tilde{\omega}_{\theta}=\omega_{\theta}\left[1-\frac{\operatorname{Re}\left(K_{s}\left(\omega_{\theta}\right)\right)}{2 K_{t}}\right] \text { and } \tilde{\gamma}=\gamma\left[1+Q \frac{\operatorname{Im}\left(K_{s}\left(\omega_{\theta}\right)\right)}{K_{t}}\right] .
$$

The modified damping and frequency of the mechanical oscillator have been estimated in the limit $K_{t} \gg \operatorname{Re}\left(K_{s}\left(\omega_{\theta}\right)\right)$, where $K_{t}=I \omega_{\theta}^{2}$ is the trap rigidity. $Q=\omega_{\theta} / \gamma$ is the quality factor of the trapped particle.

\subsubsection{Dynamical Spin-Rigidity in the Adiabatic Limit}

In the adiabatic limit $\left|\partial \rho_{10} / \partial t\right| \ll\left|\left(-\Gamma_{2}^{*}+i \Delta(\theta)\right) \rho_{10}\right|$, we have

$$
\frac{\partial \rho_{11}}{\partial t}=-\gamma_{\text {las }} \rho_{11}-\frac{\Omega^{2}}{2 \Gamma_{2}^{*}} \mathcal{L}(\theta)\left(2 \rho_{11}-1\right), \text { where } \mathcal{L}(\theta)=\frac{1}{1+\left(\Delta(\theta) / \Gamma_{2}^{*}\right)^{2}} .
$$

We now linearize this equation around the steady states and move to the Fourier space. We find:

$$
K_{s}(\omega)=\hbar N \bar{\Delta} \frac{(\alpha \tau)^{2}}{1+i \omega \tau}, \text { where } \alpha=G_{\theta} \sqrt{\frac{\gamma_{\text {las }} \Gamma_{0}^{2}}{\Gamma_{2}^{*} \Omega^{2}}} \text { and } \tau=\left(\gamma_{\text {las }}+\Gamma_{0}\right)^{-1} .
$$

Finally, using Equation (29), we obtain

$$
\tilde{\omega}_{\theta}=\omega_{\theta}\left[1+\frac{\hbar N}{2 K_{t}} \frac{(\alpha \tau)^{2}}{1+\left(\omega_{\theta} \tau\right)^{2}} \bar{\Delta}\right] \text { and } \tilde{\gamma}=\gamma\left[1-Q \frac{\hbar N(\alpha \tau)^{2}\left(\omega_{\theta} \tau\right)}{K_{t}\left(1+\left(\omega_{\theta} \tau\right)^{2}\right)} \bar{\Delta}\right]
$$


As manifest in Equation (32), when $\omega_{\theta} \tau$ is close to 1 and $\bar{\Delta}<0$, the oscillator motion can be damped, and thus cooled down. As in cavity opto-mechanics, the cooling is due to the retarded nature of the torque/force. Here, the delay comes from the finite time $\tau$ it takes to re-polarize the spins in the magnetic state after the oscillator is displaced from equilibrium, as depicted in Figure $8 \mathrm{a}$.

The change in damping induces a change in the oscillator temperature. Using $\delta \theta(\omega)=\chi_{\text {eff }}(\omega) \delta \tau_{L}(\omega)$, one finds that the variance of the angle is

$$
S_{\theta}(\omega)=\left|\chi_{\mathrm{eff}}(\omega)\right|^{2} S_{T},
$$

where $S_{T}=2 k T I \gamma$ is the two-frequency correlation of the Langevin torques. It is estimated at the temperature $T$ of the gas molecules surrounding the particle, in the regime where $\hbar \omega_{\theta} \ll k T$. Using the fluctuation dissipation theorem, one obtains a simple relation between the temperature $T_{f}$ in the presence of the $\mathrm{NV}^{-}$spins and the temperature $T$ of the gas. One indeed has $T_{f}=\frac{\gamma}{\tilde{\gamma}} T$.

For large quality factors and negative detunings, that is with a microwave tuned to the red, a pronounced cooling can take place. This effect is strongly analogous to Raman cooling or cavity cooling and was observed in [45] using diamonds levitated in a Paul trap. Figure $8 \mathrm{~b}$ shows the power-spectral density of two libration modes of a trapped diamond undergoing spin-cooling and spin-heating. The limit to the cooling efficiency will ultimately be given by back-action noise from either the radiation pressure from the laser beam that is used to polarise the spins or by the atomic spin noise. As in cavity opto-mechanics, the latter can be mitigated in the sideband resolved regime (SRR), where the trapping frequency is larger than the spin dephasing rate $\Gamma_{2}^{*}$.

The SRR regime has not been attained so far using trapped particles coupled with $\mathrm{NV}^{-}$ centers in any platform, to the best of our knowledge. Several solutions are envisioned to increase the trapping frequency and/or reduce the spin linewidth. One of them consists of coupling the NV electronic spins to the nuclear spins of the nitrogen atoms [97] to make use of the very long nuclear spin lifetime. Another solution is to employ distant coupling schemes between NV centers in ultra-pure diamonds and strongly confined ferromagnetic particles [70]. If all other heat sources occur at a rate smaller than the spin-cooling in the SRR, ground state spin-cooling of the libration or the center of mass motion can then become a reality, offering great perspectives for controlling the quantum state of the motion with the NV spin.
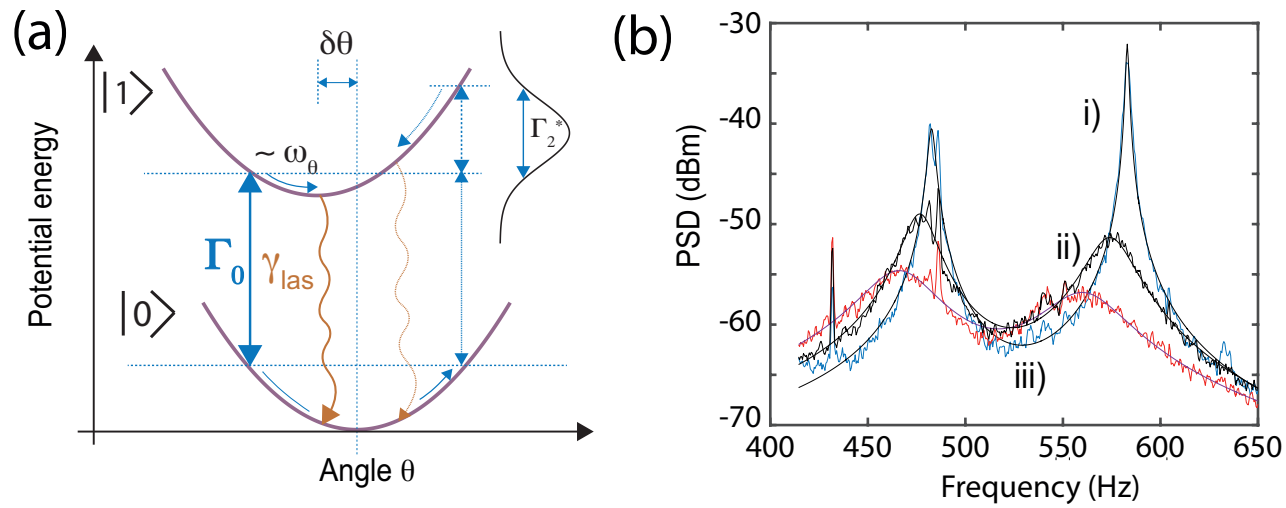

Figure 8. (a) Spin-cooling mechanism described in the adiabatic limit. The two angular potential wells in the spin states $|0\rangle$ and $|1\rangle$ are offset from one another by $\delta \theta$ due to the spin-torque. They are coupled via the laser and microwave tone at the rates $\gamma_{\text {las }}$ and $\Gamma$, respectively, see text for explanations. Here the microwave is tuned to the red, enabling cooling. (b) PSD of two librational modes when the microwave is tuned to the blue (trace i) to resonance (trace ii) and to the red (trace iii) of the spin-resonance, respectively. Adapted from [45]. 


\section{Challenges Ahead for Levitated Spin-Mechanics}

Levitated spin-mechanics offers prospects for a wide range of applications in quantum science. The negatively charge $\mathrm{NV}^{-}$center in diamond is a system of choice because of its robustness, which partly explains its increasing use in present quantum technologies. Nevertheless, recent experimental implementations have raised some technical difficulties. First and foremost, there is poor levitation stability when decreasing the gas pressure. To date, optical trapping of diamond has been reported only above mbar pressures [98], while electrodynamics and diamagnetic traps operate in the $10^{-3}$ to $10^{-4}$ mbar range $[34,36]$. To go further and operate close to the standard quantum limit for instance, the collisions with the background gas must be suppressed. Furthermore, the control of diamond in terms of shape, size, and properties is not as straightforward as for silica, for example, where chemical processes allow the production of mono-disperse spherical particles. In the present section, we highlight some of the issues that can be tackled in the next generation of levitated spin-mechanical systems.

\subsection{Production of Diamond}

The diamonds that are employed for research purposes are almost always of artificial origin. They are produced mainly using three methods, detailed in the Appendix B. The most promising technique for achieving flawless diamonds is chemical vapour deposition (CVD). In the different growth methods, the NV concentration is controlled using different strategies that depend on the initial nitrogen content in the diamond. When the sample contains large nitrogen concentrations, it can be irradiated by electrons or alpha particles to create vacancies. Diamond annealing then allows vacancy migration until being stably associated with a nitrogen atom to form a $\mathrm{NV}^{-}$center. The irradiation dose dictates the concentration of $\mathrm{NV}^{-}$centers per nano-diamond. Typically, with this technique, a $100 \mathrm{~nm}$ diamond can be doped to contain one, up to thousands of $\mathrm{NV}^{-}$centers. It has been shown that a similar result can also be obtained using laser irradiation, with the benefit of controlling the position (and the number) of the produced $\mathrm{NV}^{-}$centers [99]. When starting with pure CVD diamonds, nitrogen is first implanted and converted to $\mathrm{NV}^{-}$centers, with a few percent yield, during an annealing process.

\subsection{Control of Diamond Shape and Properties}

As discussed previously, thanks to the development of CVD grown diamonds and implantation techniques, it is possible to finely control the purity, and the number of hosted $\mathrm{NV}^{-}$centers in the diamond. Over the last decades, nano and micro-fabrication of diamond has enabled the realization of increasingly complex diamond structures [100]. Pillars of a controlled aspect-ratio can be nano-fabricated, and even more advanced structures are achievable using reactive ion etching technics. This is of particular interest for trapping diamonds with large librational frequencies [46].

The development of coated diamond initially intended for biology applications may also increase the achievable control of the properties of trapped diamond. A typical example is silica embedded diamonds, which allows obtaining spherical particles, and that have been shown to favor the $\mathrm{NV}^{-}$centers luminescence stability in optically levitated nano-diamonds [63].

Spin-mechanics with trapped objects will thus benefit from the tremendous progress in diamond material science and continuously improved knowledge about diamond and $\mathrm{NV}^{-}$centers. A potential drawback is that the production quantity of diamonds may be limited. Besides, one could be interested in levitating a specific diamond particle with physical properties that have been well-characterized beforehand. In both cases, statistical trapping procedures that start from a sprayed colloidal particle solution, and rely on random trapping events are not ideal. The different recent approaches proposed for in situ trapping, using a piezo shacking, a laser impulsion $[48,101]$, or trapping particles embedded in a polymer thin film [36], are very encouraging for the developments of on-demand trapping. 
Coupling these technics with an in situ characterization using a confocal microscope could solve these issues.

\subsection{Increasing the $\mathrm{NV}^{-}$Concentration}

We have seen that increasing the number of $\mathrm{NV}^{-}$centers that couple to the oscillator motion is a viable route towards observing strong spin-mechanical effects. While the optimal density of $\mathrm{NV}^{-}$centers is generally a compromise between sensitivity and coherence time $T_{2}^{*}$, other more exotic effects start to appear when the spin density reaches a critical point.

For concentrations that are larger than $\sim 1 \mathrm{ppm}$ (corresponding to a mean distance between spins of $\approx 15 \mathrm{~nm}$ or a dipolar coupling strength of $\approx 15 \mathrm{kHz}$ ), the dipolar coupling amongst NV spins plays an important role. One important effect is the modification of the spin lifetime $T_{1}$ through dipolar coupling with other short lived $\mathrm{NV}^{-}$centers $[66,102,103]$. This particular effect has been used with a levitating diamond in a Paul trap to observe a resonant change in the spins' magnetic susceptibility [83].

Other collective effects between $\mathrm{NV}^{-}$centers include the cooperative enhancement of the $\mathrm{NV}^{-}$centers' dipole interaction, a phenomenon similar to that of super-radiance described in [71-73], and observed with a levitating diamond in an optical tweezer by Juan et al. [74].

\subsection{Internal Temperature of Levitated Diamonds}

Numerous studies have reported an increase in levitated diamond internal temperature under vacuum conditions $[53,55,104,105]$. This heating is detrimental for practical reasons since it may lead to the burning or melting of the levitated particle. Internal temperature also induces extra quantum decoherence channels, which may prevent the observation of macroscopic quantum effects and impact the contrast of the spin resonance.

This heating was shown to be induced by laser absorption by the particle. The final temperature is the result of a competition between absorption, heat conduction to the surrounding residual gas, and black-body radiative exchange. Figure 9a shows the expected internal temperature for different diamond materials using the model detailed in references $[4,98]$. It can be seen that the expected final temperature can vary by one order of magnitude depending on the purity of the diamond material.
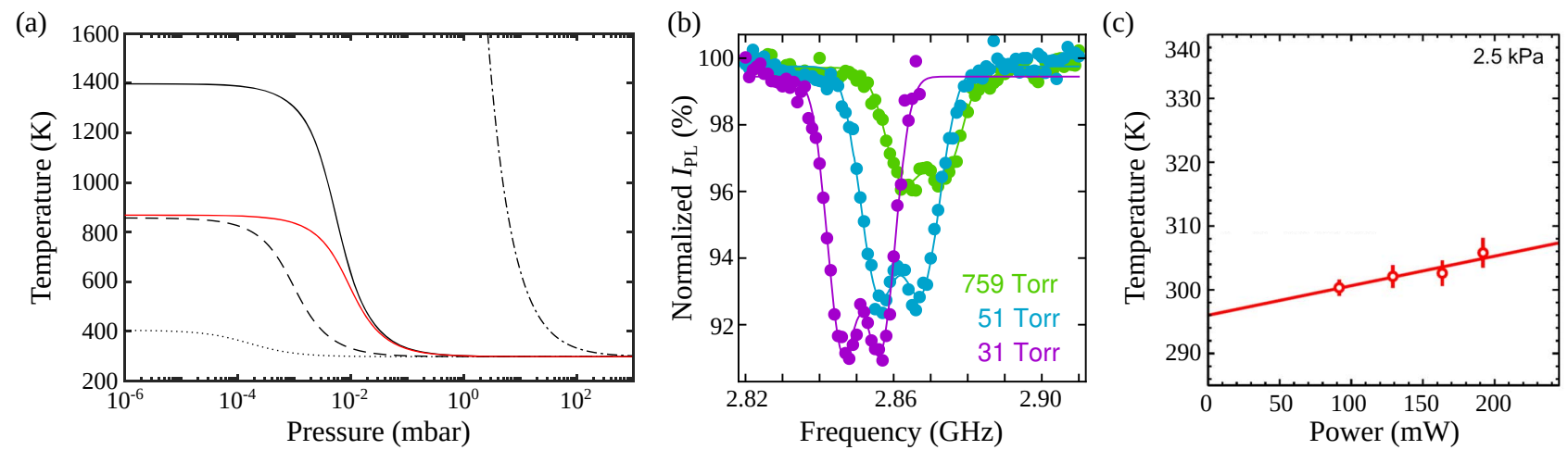

Figure 9. Internal heating of levitated diamonds. (a) Expected internal temperature of optically levitated nano-diamonds of different purity as a function of background gas pressure. The temperature dependence is shown for standard commercial diamond (---) up to the best expected grade (dotted line). The red-line corresponds to silica. Adapted from [98]. (b) Electron spin resonance from optically levitated nano-diamonds at different pressures. A clear shift of the central ESR frequency demonstrates diamond heating. Adapted from [55]. (c) Internal temperature of an optically levitated nano-diamond hosting a single $\mathrm{NV}^{-}$center as a function of trapping laser power. Adapted with permission from [53]. ( The Optical Society. 
Studies conducted on high purity CVD bulk diamonds have shown that a very low laser absorbance is achievable [106], even below the silica absorption levels. Currently however, most experiments with levitated diamonds observe orders of magnitude higher heating rates. Any defects from the diamond matrix may indeed worsen the absorption First, $\mathrm{NV}^{-}$color centers may contribute to the absorption due to non-radiative pathways of the electron population (dashed lines in Figure 9a). Most importantly, other diamond matrix defects will also play their role, from isolated nitrogen atoms to graphite on the diamond surface, through grain boundary or other atomic impurities [107].

The spin of the $\mathrm{NV}^{-}$centers is actually an invaluable tool to estimate the diamond temperature $[55,104,108]$ because the zero-field splitting $D$, between $m_{s}=0$ and $m_{s}= \pm 1$ states is temperature dependent, and well characterized [109]. One can measure the internal temperature of a levitated diamond by measuring the ESR of the $\mathrm{NV}^{-}$centers it hosts $[55,104]$, see Figure 9b,c which shows heating measurements from trapped diamonds under vacuum.

Note that instead of improving the properties of trapped diamonds that are destined to be trapped under a magnetic field produced by a fixed magnet, the roles of the diamond and the magnet can be reversed. Schemes already discussed in this review [35,70] involving trapped magnets were recently proposed. In these proposals, the diamond containing $\mathrm{NV}^{-}$ centers is attached to a large heat sink, so it does not heat up significantly and can readily be made from CVD for improved spin-properties. Furthermore, no strong laser needs to be shone onto the trapped magnet.

\subsection{Beyond $N V^{-}$Centers and Diamond}

The present review focused on $\mathrm{NV}^{-}$centers in diamond, which is by far the most studied system for levitated spin-mechanics. However, our discussions may be applied to other color centers with similar behavior, and also solve some of the issues discussed in this section. Typically, over recent decades, color centers in $\mathrm{SiC}$ have been shown to own an optically addressable spin resonance [110]. SiC can benefit from silicon-like technologies, which offer a good level of control over the material and its nano fabrication. Ultimately, with the recent isolation of single color centers [111], even silicon may become an excellent platform for spin-mechanics.

\section{Conclusions}

In this review, we presented recent levitated spin-mechanics experiments, focusing specifically on $\mathrm{NV}^{-}$centers in diamonds. We introduced a formalism describing the spin-mechanical interactions in these experiments and highlighted the advantages and limitations of this interaction scheme. We discussed the technical challenges that remain towards taking the full benefit of levitated spin-mechanics. The common goals and emulation from atomic and solid-state physic, material science, and the levitation scientific communities is a cornerstone for success in this field. This will undoubtedly lead to innovative and exciting experiments and applications.

Author Contributions: Conceptualization, G.H., L.R. and M.P.; methodology, G.H., M.P., P.H. and L.R.; formal analysis, G.H. and M.P; writing-review and editing, G.H., L.R., C.P.-M. and M.P. All authors have read and agreed to the published version of the manuscript.

Funding: This research was funded by the Île-de-France Region in the framework of DIM SIRTEQ and by the Investissements d'Avenir of LabEx PALM (ANR-10-LABX-0039-PALM).

Acknowledgments: We would like to acknowledge fruitful discussions with Samuel Deléglise, Itsik Cohen, Cosimo Rusconi, Oriol Romero-Isart and Benjamin Stickler and thank James Millen for proof reading the manuscript.

Conflicts of Interest: The authors declare no conflict of interest. 


\section{Abbreviations}

The following abbreviations are used in this manuscript:

ESR Electron spin resonance

ODMR Optically detected magnetic resonance

CVD Chemical Vapor Deposition

PSD Power spectral density

\section{Appendix A. Derivation of a Simplified Hamiltonian}

In this section, we detail the calculation of the simplified spin-libration Hamiltonian Equation (13). Several unitary transformations will be performed on the Hamiltonian. For the sake of clarity, we will divide our Hamiltonian into three different parts to do the calculation and see the effect of each transformation on the different parts. We write

$$
\hat{\mathcal{H}}=\hat{\mathcal{H}}_{\text {mecha }}+\hat{\mathcal{H}}_{\mathrm{NV}}+\hat{\mathcal{H}}_{\mu \mathrm{w}}
$$

where

$$
\begin{gathered}
\hat{\mathcal{H}}_{\text {mecha }}=\frac{\hat{p}_{\theta}^{2}}{2 I}+\frac{1}{2} I \omega_{\theta}^{2}\left(\hat{\theta}-\theta^{\prime}\right)^{2}, \\
\hat{\mathcal{H}}_{\mathrm{NV}}=\hbar D \hat{S}_{z^{\prime}}^{2}+\hbar \gamma_{e} B \hat{S}_{z}
\end{gathered}
$$

and

$$
\hat{\mathcal{H}}_{\mu \mathrm{w}}=\hbar \Omega \cos (\omega t) \hat{S}_{x}
$$

A few approximations have been made to derive this Hamiltonian. We suppose that $\gamma_{e} B$ is smaller than the zero-field splitting $D$ of the $\mathrm{NV}^{-}$center. We suppose the transverse magnetic field $B_{\perp}$ and the longitudinal magnetic field $B_{\|}$to be of the same order of magnitude. We also suppose that the angular momentum of the particle, given by the mean value $\left\langle\hat{p}_{\theta}\right\rangle$, is a few order of magnitude larger than the typical spin momenta $\hbar N\left\langle\hat{S}_{y}\right\rangle$. This assumption is valid for micron-sized particles. Finally, we consider that $\Omega \ll \gamma_{e} B$.

\section{Appendix A.1. Moving to the Particle Frame}

We move to the particle frame by performing the unitary transformation $\hat{U}=e^{i \hat{\theta} \hat{S}_{y}}$. This frame is relevant because the eigenstates of the new spin operator $\hat{S}_{z}$ are now the ones where the optical pumping process of the green laser takes place. In this frame, the $\mathrm{NV}^{-}$ Hamiltonian reads:

$$
\hat{\mathcal{H}}_{\mathrm{NV}}^{\prime}=\hbar D \hat{S}_{z}^{2}+\hbar \gamma_{e} B\left(\cos \hat{\theta} \hat{S}_{z}-\sin \hat{\theta} \hat{S}_{x}\right)
$$

The mechanical part of the Hamiltonian becomes

$$
\hat{\mathcal{H}}_{\text {mecha }}^{\prime}=\frac{\left(\hat{p}_{\theta}-\hbar \hat{S}_{y}\right)^{2}}{2 I}+\frac{1}{2} I \omega_{\theta}^{2}\left(\hat{\theta}-\theta^{\prime}\right)^{2} .
$$

One of the assumption that we have made is to neglect the spin contribution to the total angular momenta of the system, which means $\hbar N\left\langle\hat{S}_{y}\right\rangle \ll\left\langle\hat{p}_{\theta}\right\rangle$. Thus, we have:

$$
\hat{\mathcal{H}}_{\text {mecha }}^{\prime} \simeq \frac{\hat{p}_{\theta}^{2}}{2 I}+\frac{1}{2} I \omega_{\theta}^{2}\left(\hat{\theta}-\theta^{\prime}\right)^{2} .
$$

The microwave part of the Hamiltonian simply becomes

$$
\hat{\mathcal{H}}_{\mu \mathrm{w}}^{\prime}=\hbar \Omega \cos (\omega t)\left(\cos \hat{\theta} \hat{S}_{x}+\sin \hat{\theta} \hat{S}_{z}\right) .
$$




\section{Appendix A.2. Diagonalization of the $N V^{-}$Hamiltonian}

The $\mathrm{NV}^{-}$part of the Hamiltonian is diagonalized in the perturbative limit $\frac{\gamma_{e} B}{D} \ll 1$. We introduce the operators $\hat{u}_{\perp}=\frac{\gamma_{e} B}{D} \sin \hat{\theta}$ and $\hat{u}_{\|}=\frac{\gamma_{e} B}{D} \cos \hat{\theta}$. Thus, we have $\left\langle\hat{u}_{\perp}\right\rangle \ll 1$ and $\left\langle\hat{u}_{\|}\right\rangle \ll 1$. Using these operators, the Hamiltonian of the NV reads:

$$
\hat{\mathcal{H}}_{\mathrm{NV}}^{\prime}=\hbar D \hat{S}_{z}^{2}+\hbar D\left(\hat{u}_{\|} \hat{S}_{z}-\hat{u}_{\perp} \hat{S}_{x}\right)
$$

We can treat the second part of this Hamiltonian as a perturbation and move to the basis where this Hamiltonian is diagonal to second order in $\frac{\gamma_{e} B}{D}$. We consider the unitary transformation:

$$
\hat{U}^{\prime \prime}=\left(\begin{array}{ccc}
1-\frac{\hat{u}_{\perp}^{2}}{4} & \frac{\hat{u}_{\perp}}{\sqrt{2}}\left(1-\hat{u}_{\|}\right) & -\frac{\hat{u}_{\perp}^{2}}{4} \\
-\frac{\hat{u}_{\perp}}{\sqrt{2}}\left(1-\hat{u}_{\|}\right) & 1-\frac{\hat{u}_{\perp}^{2}}{2} & -\frac{\hat{u}_{\perp}}{\sqrt{2}}\left(1+\hat{u}_{\|}\right) \\
-\frac{\hat{u}_{\perp}^{2}}{4} & \frac{\hat{u}_{\perp}}{\sqrt{2}}\left(1+\hat{u}_{\|}\right) & 1-\frac{\hat{u}_{\perp}^{2}}{4}
\end{array}\right) .
$$

The new states we are considering by applying this transformation are defined by

$$
\begin{aligned}
\left|+1^{\prime}\right\rangle & =\left(1-\frac{\hat{u}_{\perp}^{2}}{4}\right)|+1\rangle-\frac{\hat{u}_{\perp}}{\sqrt{2}}\left(1-\hat{u}_{\|}\right)|0\rangle-\frac{\hat{u}_{\perp}^{2}}{4}|-1\rangle \\
\left|0^{\prime}\right\rangle & =\frac{\hat{u}_{\perp}}{\sqrt{2}}\left(1-\hat{u}_{\|}\right)|+1\rangle+\left(1-\frac{\hat{u}_{\perp}^{2}}{2}\right)|0\rangle+\frac{\hat{u}_{\perp}}{\sqrt{2}}\left(1+\hat{u}_{\|}\right)|-1\rangle \\
\left|-1^{\prime}\right\rangle & =-\frac{\hat{u}_{\perp}^{2}}{4}|+1\rangle-\frac{\hat{u}_{\perp}}{\sqrt{2}}\left(1+\hat{u}_{\|}\right)|0\rangle+\left(1-\frac{\hat{u}_{\perp}^{2}}{4}\right)|-1\rangle .
\end{aligned}
$$

We have $\hat{U}^{\prime \prime \dagger} \hat{U}^{\prime \prime}=\hat{I} d+o\left(\left\|\left(\hat{u}_{\perp}, \hat{u}_{\|}\right)\right\|^{2}\right)$ which means that $\hat{U}^{\prime \prime}$ is unitary up to second order in $\left\|\left(\hat{u}_{\perp}, \hat{u}_{\|}\right)\right\|$. Under this transformation, the NV Hamiltonian reads

$$
\hat{\mathcal{H}}_{\mathrm{NV}}^{\prime \prime} \simeq \hbar D\left(\begin{array}{ccc}
1+\hat{u}_{\|}+\frac{\hat{u}_{\perp}^{2}}{2} & 0 & \frac{\hat{u}_{\perp}^{2}}{2} \\
0 & -\hat{u}_{\perp}^{2} & 0 \\
\frac{\hat{u}_{\perp}^{2}}{2} & 0 & 1-\hat{u}_{\|}+\frac{\hat{u}_{\perp}^{2}}{2}
\end{array}\right)
$$

which can be written as:

$$
\hat{\mathcal{H}}_{\mathrm{NV}}^{\prime \prime} \simeq \hbar D\left(\begin{array}{ccc}
1+\frac{\gamma_{e} B}{D} \cos \hat{\theta}+\left(\frac{\gamma_{C} B}{D}\right)^{2} \frac{\sin \hat{\theta}^{2}}{2} & 0 & \left(\frac{\gamma_{C} B}{D}\right)^{2} \frac{\sin \hat{\theta}^{2}}{2} \\
0 & -\left(\frac{\gamma_{e} B}{D}\right)^{2} \sin \hat{\theta}^{2} & 0 \\
\left(\frac{\gamma_{C} B}{D}\right)^{2} \frac{\sin \hat{\theta}^{2}}{2} & 0 & 1-\frac{\gamma_{C} B}{D} \cos \hat{\theta}+\left(\frac{\gamma_{e} B}{D}\right)^{2} \frac{\sin \hat{\theta}^{2}}{2}
\end{array}\right) .
$$

Furthermore, we suppose that the angle satisfies $\left\langle\hat{u}_{\perp}\right\rangle \simeq\left\langle\hat{u}_{\|}\right\rangle$which implies that $\left(\frac{\gamma_{e} B}{D}\right)^{2} \frac{\langle\sin \hat{\theta}\rangle^{2}}{2} \ll \frac{\gamma_{e} B}{D}\langle\cos \hat{\theta}\rangle$. We can then neglect non diagonal terms in this regime and we obtain a diagonal Hamiltonian:

$$
\hat{\mathcal{H}}_{\mathrm{NV}}^{\prime \prime} \simeq \hbar D\left(\begin{array}{ccc}
1+\frac{\gamma_{e} B}{D} \cos \hat{\theta}+\left(\frac{\gamma_{e} B}{D}\right)^{2} \frac{\sin \hat{\theta}^{2}}{2} & 0 & 0 \\
0 & -\left(\frac{\gamma_{e} B}{D}\right)^{2} \sin \hat{\theta}^{2} & 0 \\
0 & 0 & 1-\frac{\gamma_{e} B}{D} \cos \hat{\theta}+\left(\frac{\gamma_{e} B}{D}\right)^{2} \frac{\sin \hat{\theta}^{2}}{2}
\end{array}\right) .
$$

Let us apply this transformation to the mechanical part of the Hamiltonian. The unitary transformation $\hat{U}^{\prime \prime}$ only depends on $\hat{\theta}$ so it commutes with it. This transformation can be written $\hat{U}^{\prime \prime}=\hat{I} d+u \hat{V}(\hat{\theta})+o(|u|)$ with $u=\frac{\gamma_{e} B}{D}$ and $\hat{V}(\hat{\theta})=\hat{A} \sin \hat{\theta}$ where 
$\hat{A}=\frac{1}{\sqrt{2}}(|+1\rangle\langle 0|-| 0\rangle\langle+1|+|-1\rangle\langle 0|-| 0\rangle\langle-1|)$. Furthermore, we have $\hat{V}(\hat{\theta})^{\dagger}=-\hat{V}(\hat{\theta})$. We have:

$$
\begin{gathered}
\hat{U}^{\prime \prime \dagger} \hat{p}_{\theta} \hat{U}^{\prime \prime}=(\hat{I} d-u \hat{V}(\hat{\theta})+o(|u|)) \hat{p}_{\theta}(\hat{I} d+u \hat{V}(\hat{\theta})+o(|u|)) \\
\hat{U}^{\prime \prime \dagger} \hat{p}_{\theta} \hat{U}^{\prime \prime}=\hat{p}_{\theta}+u\left[\hat{p}_{\theta}, \hat{V}(\hat{\theta})\right]+o(|u|) \\
\hat{U}^{\prime \prime \dagger} \hat{p}_{\theta} \hat{U}^{\prime \prime}=\hat{p}_{\theta}+u \hat{A}\left[\hat{p}_{\theta}, \sin \hat{\theta}\right]+o(|u|) \\
\hat{U}^{\prime \prime \dagger} \hat{p}_{\theta} \hat{U}^{\prime \prime}=\hat{p}_{\theta}-i \hbar u \hat{A} \cos \hat{\theta}+o(|u|) .
\end{gathered}
$$

We can safely neglect the second term under the initial assumption $\left\langle\hat{p}_{\theta}\right\rangle \gg \hbar N u$. Thus, we get:

$$
\hat{\mathcal{H}}_{\text {mecha }}^{\prime \prime} \simeq \frac{\hat{p}_{\theta}^{2}}{2 I}+\frac{1}{2} I \omega_{\theta}^{2}\left(\hat{\theta}-\theta^{\prime}\right)^{2} .
$$

Furthermore, this transformation does not affect the microwave Hamiltonian to first order in $\frac{\gamma_{e} B}{D}$ so we get:

$$
\hat{\mathcal{H}}_{\mu \mathrm{w}}^{\prime \prime} \simeq \hbar \Omega \cos (\omega t)\left(\cos \hat{\theta} \hat{S}_{x}+\sin \hat{\theta} \hat{S}_{z}\right)
$$

\section{Appendix A.3. Equilibrium Position of the Paul Trap}

We apply the unitary transformation $\hat{U}^{\prime \prime \prime}=e^{i \theta^{\prime}} \hat{p}_{\theta} / \hbar$ which redefines $\hat{\theta}$ as $\hat{\theta}-\theta^{\prime}$. This transformation does not affect the eigenstates of the spin and we obtain to first order in $\hat{\theta}$ :

$$
\hat{\mathcal{H}}_{\mathrm{NV}}^{\prime \prime \prime} \simeq \hbar\left(\omega_{+1}+\beta_{+1} \hat{\theta}\right)\left|+1^{\prime}\right\rangle\left\langle+1^{\prime}\left|+\hbar\left(\omega_{0}+\beta_{0} \hat{\theta}\right)\right| 0^{\prime}\right\rangle\left\langle 0^{\prime}\left|+\hbar\left(\omega_{-1}+\beta_{-1} \hat{\theta}\right)\right|-1^{\prime}\right\rangle\left\langle-1^{\prime}\right|,
$$

with

$$
\begin{aligned}
\omega_{+1} & =D+\gamma_{e} B \cos \left(\theta^{\prime}\right)+\frac{\left(\gamma_{e} B\right)^{2}}{D} \frac{\sin \left(\theta^{\prime}\right)^{2}}{2} \\
\omega_{0} & =-\frac{\left(\gamma_{e} B\right)^{2}}{D} \sin \left(\theta^{\prime}\right)^{2} \\
\omega_{-1} & =D-\gamma_{e} B \cos \left(\theta^{\prime}\right)+\frac{\left(\gamma_{e} B\right)^{2}}{D} \frac{\sin \left(\theta^{\prime}\right)^{2}}{2} \\
\beta_{i} & =\frac{\partial \omega_{i}}{\partial \theta^{\prime}} .
\end{aligned}
$$

This transformation shifts the equilibrium position of the mechanical oscillator by $\theta^{\prime}$ and we get:

$$
\hat{\mathcal{H}}_{\text {mecha }}^{\prime \prime \prime} \simeq \frac{\hat{p}_{\theta}^{2}}{2 I}+\frac{1}{2} I \omega_{\theta}^{2} \hat{\theta}^{2} .
$$

The microwave Hamiltonian reads

$$
\hat{\mathcal{H}}_{\mu \mathrm{w}}^{\prime \prime \prime} \simeq \hbar \Omega \cos (\omega t)\left(\cos \left(\theta^{\prime}+\hat{\theta}\right) \hat{S}_{x}+\sin \left(\theta^{\prime}+\hat{\theta}\right) \hat{S}_{z}\right) .
$$

\section{Appendix A.4. Rotating Frame of the Micro-Wave}

The last unitary transformation is to move to the microwave frame by performing the unitary transformation $\hat{U}^{\prime \prime \prime \prime}=e^{i \omega t \hat{S}_{z}^{2}}$. This transformation is diagonal so it commutes with 
the $\mathrm{NV}^{-}$center Hamiltonian which is also diagonal. As it is a time-dependent transformation, this will add a shift in energy to both the $\left|+1^{\prime}\right\rangle$ and $\left|-1^{\prime}\right\rangle$ states which gives the Hamiltonian:

$$
\begin{aligned}
\hat{\mathcal{H}}_{\mathrm{NV}}^{\prime \prime \prime \prime} & \simeq \hbar\left(-\Delta_{+1}+\beta_{+1} \hat{\theta}\right)\left|+1^{\prime}\right\rangle\left\langle+1^{\prime}\left|+\hbar\left(-\Delta_{0}+\beta_{0} \hat{\theta}\right)\right| 0^{\prime}\right\rangle\left\langle 0^{\prime}\right| \\
& +\hbar\left(-\Delta_{-1}+\beta_{-1} \hat{\theta}\right)\left|-1^{\prime}\right\rangle\left\langle-1^{\prime}\right|,
\end{aligned}
$$

by defining $\Delta_{+1}=\omega-\omega_{+1}, \Delta_{-1}=\omega-\omega_{-1}$ and $\Delta_{0}=-\omega_{0}$.

The mechanical Hamiltonian is not affected by this transformation as it commutes with it. Finally, under the rotating wave approximation and by neglecting first order terms in $\hat{\theta}$ which are negligible since $\Omega \ll \gamma_{e} B$, we obtain:

$$
\hat{\mathcal{H}}_{\mu \mathrm{w}}^{\prime \prime \prime \prime} \simeq \hbar \frac{\Omega}{2} \cos \left(\theta^{\prime}\right) \hat{S}_{x}
$$

Redefining $\Omega=\Omega \cos \left(\theta^{\prime}\right)$, we get:

$$
\hat{\mathcal{H}}_{\mu \mathrm{w}}^{\prime \prime \prime \prime} \simeq \hbar \frac{\Omega}{2} \hat{S}_{x} .
$$

Finally, the Hamiltonian can be simply written as:

$$
\hat{\mathcal{H}}^{\prime \prime \prime \prime}=\hat{\mathcal{H}}_{\text {mecha }}^{\prime \prime \prime \prime}+\hat{\mathcal{H}}_{\mathrm{NV}}^{\prime \prime \prime \prime}+\hat{\mathcal{H}}_{\mu \mathrm{w}}^{\prime \prime \prime \prime}
$$

where

$$
\begin{gathered}
\hat{\mathcal{H}}_{\text {mecha }}^{\prime \prime \prime \prime}=\frac{\hat{p}_{\theta}^{2}}{2 I}+\frac{1}{2} I \omega_{\theta}^{2} \hat{\theta}^{2} . \\
\hat{\mathcal{H}}_{\mathrm{NV}}^{\prime \prime \prime \prime} \simeq \hbar\left(-\Delta_{+1}+\beta_{+1} \hat{\theta}\right)\left|+1^{\prime}\right\rangle\left\langle+1^{\prime}\left|+\hbar\left(-\Delta_{0}+\beta_{0} \hat{\theta}\right)\right| 0^{\prime}\right\rangle\left\langle 0^{\prime}\left|+\hbar\left(-\Delta_{-1}+\beta_{-1} \hat{\theta}\right)\right|-1^{\prime}\right\rangle\left\langle-1^{\prime}\right|
\end{gathered}
$$

and

$$
\hat{\mathcal{H}}_{\mu \mathrm{w}}^{\prime \prime \prime \prime} \simeq \hbar \frac{\Omega}{2} \hat{S}_{x}
$$

\section{Appendix B. Diamond Synthesis}

There are three main diamond synthesis methods:

- The HPHT process (high pressure, high temperature): A carbon precursor is brought under conditions of high pressure (typically $>5 \mathrm{GPa}$ ) and high temperature $\left(\mathrm{T} \approx 2000^{\circ} \mathrm{C}\right)$ in order to create diamond. While this approach has been known since the 1950s, the control of impurities in the diamond is not straigthforward. The diamonds produced are often rich in nitrogen impurities, typically around 200 ppm. Most recent works on diamond levitation used HPHT diamonds due to their ease of use and commercial availability.

- The CVD growth (chemical vapor deposition). A reactor is used to deposit carbon atoms from a methane gas, layer by layer on a diamond substrate. It is then possible to finely control the impurities present in the diamond. It is the method of choice to create diamonds with very high purity. The concentration of paramagnetic species such a nitrogen or silicon can indeed be reduced below the detection level, and the concentration of ${ }^{13} \mathrm{C}$ atoms below natural abundance. Importantly, CVD growth also enables $\mathrm{NV}^{-}$center doping at any time during the growth [112].

- Detonation nanodiamonds are obtained by an explosive reaction from a carbon precursor. This approach provides very small nanodiamonds, typically $<10 \mathrm{~nm}$, which are often highly graphitized [113]. Such diamonds are thus not suited for the applications discussed in the present review. 
Although recent efforts towards micro or nano-diamond creation have been made towards direct CVD [114,115] and HPHT [116] synthesis, the general strategy is to mill bulk diamonds in order to obtain nano (and even micro) diamonds. The size of the diamonds is then selected by centrifugation.

\section{References}

1. Aspelmeyer, M.; Kippenberg, T.J.; Marquardt, F. Cavity optomechanics. Rev. Mod. Phys. 2014, 86, 1391-1452. [CrossRef]

2. Millen, J.; Monteiro, T.S.; Pettit, R.; Vamivakas, A.N. Optomechanics with levitated particles. Rep. Prog. Phys. $2020,83,026401$. [CrossRef]

3. Hebestreit, E.; Frimmer, M.; Reimann, R.; Novotny, L. Sensing Static Forces with Free-Falling Nanoparticles. Phys. Rev. Lett. 2018, 121, 063602. [CrossRef]

4. Chang, D.E.; Regal, C.A.; Papp, S.B.; Wilson, D.J.; Ye, J.; Painter, O.; Kimble, H.J.; Zoller, P. Cavity Opto-Mechanics Using an Optically Levitated Nanosphere. Proc. Natl. Acad. Sci. USA 2010, 107, 1005-1010. [CrossRef]

5. Romero-Isart, O.; Juan, M.L.; Quidant, R.; Cirac, J.I. Toward Quantum Superposition of Living Organisms. New J. Phys. 2010, 12, 033015. [CrossRef]

6. Barker, P.F.; Shneider, M.N. Cavity cooling of an optically trapped nanoparticle. Phys. Rev. A 2010, 81, 023826. [CrossRef]

7. Delić, U.; Reisenbauer, M.; Dare, K.; Grass, D.; Vuletić, V.; Kiesel, N.; Aspelmeyer, M. Motional Quantum Ground State of a Levitated Nanoparticle from Room Temperature. arXiv 2019, arXiv:191104406.

8. Tebbenjohanns, F.; Frimmer, M.; Jain, V.; Windey, D.; Novotny, L. Motional Sideband Asymmetry of a Nanoparticle Optically Levitated in Free Space. Phys. Rev. Lett. 2020, 124, 013603. [CrossRef] [PubMed]

9. Magrini, L.; Rosenzweig, P.; Bach, C.; Deutschmann-Olek, A.; Hofer, S.G.; Hong, S.; Kiesel, N.; Kugi, A.; Aspelmeyer, M. Optimal Quantum Control of Mechanical Motion at Room Temperature: Ground-State Cooling. arXiv 2020, arXiv:201215188.

10. Tebbenjohanns, F.; Mattana, M.L.; Rossi, M.; Frimmer, M.; Novotny, L. Quantum control of a nanoparticle optically levitated in cryogenic free space. arXiv 2021, arXiv:2103.03853.

11. Moore, D.C.; Geraci, A.A. Searching for New Physics Using Optically Levitated Sensors. arXiv 2020, arXiv:200813197.

12. Xiong, F.; Wu, T.; Leng, Y.; Li, R.; Duan, C.; Kong, X.; Huang, P.; Li, Z.; Gao, Y.; Rong, X.; et al. Searching Spin-Mass Interaction Using a Diamagnetic Levitated Magnetic Resonance Force Sensor. arXiv 2020, arXiv:201014199.

13. Vinante, A.; Carlesso, M.; Bassi, A.; Chiasera, A.; Varas, S.; Falferi, P.; Margesin, B.; Mezzena, R.; Ulbricht, H. Narrowing the Parameter Space of Collapse Models with Ultracold Layered Force Sensors. Phys. Rev. Lett. 2020, 125, 100404. [CrossRef] [PubMed]

14. Rabl, P.; Cappellaro, P.; Dutt, M.V.G.; Jiang, L.; Maze, J.R.; Lukin, M.D. Strong magnetic coupling between an electronic spin qubit and a mechanical resonator. Phys. Rev. B 2009, 79, 041302. [CrossRef]

15. Treutlein, P.; Genes, C.; Hammerer, K.; Poggio, M.; Rabl, P. Hybrid Mechanical Systems. In Cavity Optomechanics: Nano- and Micromechanical Resonators Interacting with Light; Aspelmeyer, M., Kippenberg, T.J., Marquardt, F., Eds.; Springer: Berlin/Heidelberg, Germany, 2014; pp. 327-351. [CrossRef]

16. Arcizet, O.; Jacques, V.; Siria, A.; Poncharal, P.; Vincent, P.; Seidelin, S. A single nitrogen-vacancy defect coupled to a nanomechanical oscillator. Nat. Phys. 2011, 7, 879-883. [CrossRef]

17. Kolkowitz, S.; Bleszynski Jayich, A.C.; Unterreithmeier, Q.P.; Bennett, S.D.; Rabl, P.; Harris, J.G.E.; Lukin, M.D. Coherent Sensing of a Mechanical Resonator with a Single-Spin Qubit. Science 2012, 335, 1603-1606. [CrossRef] [PubMed]

18. Yin, Z.; Zhao, N.; Li, T. Hybrid opto-mechanical systems with nitrogen-vacancy centers. Sci. China Phys. Mech. Astron. 2015, 58, 1-12. [CrossRef]

19. Ma, Y.; Hoang, T.M.; Gong, M.; Li, T.; Yin, Z.Q. Proposal for quantum many-body simulation and torsional matter-wave interferometry with a levitated nanodiamond. Phys. Rev. A 2017, 96, 023827. [CrossRef]

20. Wei, B.B.; Burk, C.; Wrachtrup, J.; Liu, R.B. Magnetic ordering of nitrogen-vacancy centers in diamond via resonator-mediated coupling. EPJ Quantum Technol. 2015, 2, 18. [CrossRef]

21. Scala, M.; Kim, M.S.; Morley, G.W.; Barker, P.F.; Bose, S. Matter-Wave Interferometry of a Levitated Thermal Nano-Oscillator Induced and Probed by a Spin. Phys. Rev. Lett. 2013, 111, 180403. [CrossRef]

22. Pedernales, J.S.; Morley, G.W.; Plenio, M.B. Motional Dynamical Decoupling for Interferometry with Macroscopic Particles. Phys. Rev. Lett. 2020, 125, 023602. [CrossRef]

23. Rabl, P.; Kolkowitz, S.J.; Koppens, F.H.L.; Harris, J.G.E.; Zoller, P.; Lukin, M.D. A quantum spin transducer based on nanoelectromechanical resonator arrays. Nat. Phys. 2010, 6, 602. [CrossRef]

24. Martinetz, L.; Hornberger, K.; Stickler, B.A. Gas-Induced Friction and Diffusion of Rigid Rotors. Phys. Rev. E 2018, $97,052112$. [CrossRef] [PubMed]

25. Gieseler, J.; Novotny, L.; Quidant, R. Thermal Nonlinearities in a Nanomechanical Oscillator. Nat. Phys. 2013, 9, 806-810. [CrossRef]

26. Ashkin, A. Optical trapping and manipulation of neutral particles using lasers. Proc. Natl. Acad. Sci. USA 1997, 94, 4853-4860. [CrossRef] [PubMed]

27. Jones, P.H.; Marag, O.M.; Volpe, G. Optical Tweezers: Principles and Applications, 1st ed.; Cambridge University Press: Cambridge, UK, 2016. 
28. Gieseler, J.; Deutsch, B.; Quidant, R.; Novotny, L. Subkelvin Parametric Feedback Cooling of a Laser-Trapped Nanoparticle. Phys. Rev. Lett. 2012, 109, 103603. [CrossRef] [PubMed]

29. Kiesel, N.; Blaser, F.; Delić, U.; Grass, D.; Kaltenbaek, R.; Aspelmeyer, M. Cavity Cooling of an Optically Levitated Submicron Particle. Proc. Natl. Acad. Sci. USA 2013, 110, 14180-14185. [CrossRef]

30. Magrini, L.; Norte, R.A.; Riedinger, R.; Marinković, I.; Grass, D.; Delić, U.; Gröblacher, S.; Hong, S.; Aspelmeyer, M. Near-Field Coupling of a Levitated Nanoparticle to a Photonic Crystal Cavity. Optica 2018, 5, 1597-1602. [CrossRef]

31. Paul, W. Electromagnetic traps for charged and neutral particles. Rev. Mod. Phys. 1990, 62, 531-540. [CrossRef]

32. Dania, L.; Bykov, D.S.; Knoll, M.; Mestres, P.; Northup, T.E. Optical and electrical feedback cooling of a silica nanoparticle levitated in a Paul trap. Phys. Rev. Res. 2021, 3, 013018. [CrossRef]

33. Nagornykh, P.; Coppock, J.E.; Kane, B.E. Cooling of levitated graphene nanoplatelets in high vacuum. Appl. Phys. Lett. 2015, 106, 244102. [CrossRef]

34. Conangla, G.P.; Schell, A.W.; Rica, R.A.; Quidant, R. Motion Control and Optical Interrogation of a Levitating Single Nitrogen Vacancy in Vacuum. Nano Lett. 2018, 18, 3956-3961. [CrossRef]

35. Gieseler, J.; Kabcenell, A.; Rosenfeld, E.; Schaefer, J.D.; Safira, A.; Schuetz, M.J.A.; Gonzalez-Ballestero, C.; Rusconi, C.C.; Romero-Isart, O.; Lukin, M.D. Single-Spin Magnetomechanics with Levitated Micromagnets. Phys. Rev. Lett. 2020, $124,163604$. [CrossRef] [PubMed]

36. Hsu, J.F.; Ji, P.; Dutt, M.V.G.; D’Urso, B.R. Loading an Optical Trap with Diamond Nanocrystals Containing Nitrogen-Vacancy Centers from a Surface. arXiv 2015, arXiv:150608215.

37. O'Brien, M.C.; Dunn, S.; Downes, J.E.; Twamley, J. Magneto-mechanical trapping of micro-diamonds at low pressures. Appl. Phys. Lett. 2019, 114, 053103. [CrossRef]

38. Arita, Y.; Mazilu, M.; Dholakia, K. Laser-Induced Rotation and Cooling of a Trapped Microgyroscope in Vacuum. Nat. Commun. 2013, 4, 2374. [CrossRef] [PubMed]

39. Reimann, R.; Doderer, M.; Hebestreit, E.; Diehl, R.; Frimmer, M.; Windey, D.; Tebbenjohanns, F.; Novotny, L. GHz Rotation of an Optically Trapped Nanoparticle in Vacuum. Phys. Rev. Lett. 2018, 121, 033602. [CrossRef] [PubMed]

40. Monteiro, F.; Ghosh, S.; van Assendelft, E.C.; Moore, D.C. Optical Rotation of Levitated Spheres in High Vacuum. Phys. Rev. A 2018, 97, 051802. [CrossRef]

41. Ahn, J.; Xu, Z.; Bang, J.; Deng, Y.H.; Hoang, T.M.; Han, Q.; Ma, R.M.; Li, T. Optically Levitated Nanodumbbell Torsion Balance and GHz Nanomechanical Rotor. Phys. Rev. Lett. 2018, 121, 033603. [CrossRef]

42. Barnett, S.J. Gyromagnetic and Electron-Inertia Effects. Rev. Mod. Phys. 1935, 7, 129-166. [CrossRef]

43. Hoang, T.M.; Ma, Y.; Ahn, J.; Bang, J.; Robicheaux, F.; Yin, Z.Q.; Li, T. Torsional Optomechanics of a Levitated Nonspherical Nanoparticle. Phys. Rev. Lett. 2016, 117, 123604. [CrossRef]

44. Vinante, A.; Falferi, P.; Gasbarri, G.; Setter, A.; Timberlake, C.; Ulbricht, H. Ultralow Mechanical Damping with Meissner-Levitated Ferromagnetic Microparticles. Phys. Rev. Appl. 2020, 13, 064027. [CrossRef]

45. Delord, T.; Huillery, P.; Nicolas, L.; Hétet, G. Spin-Cooling of the Motion of a Trapped Diamond. Nature 2020, 1-4. [CrossRef]

46. Delord, T.; Nicolas, L.; Chassagneux, Y.; Hétet, G. Strong Coupling between a Single Nitrogen-Vacancy Spin and the Rotational Mode of Diamonds Levitating in an Ion Trap. Phys. Rev. A 2017, 96, 063810. [CrossRef]

47. Ranjit, G.; Montoya, C.; Geraci, A.A. Cold Atoms as a Coolant for Levitated Optomechanical Systems. Phys. Rev. A 2015, 91, 013416. [CrossRef]

48. Bykov, D.S.; Mestres, P.; Dania, L.; Schmöger, L.; Northup, T.E. Direct Loading of Nanoparticles under High Vacuum into a Paul Trap for Levitodynamical Experiments. Appl. Phys. Lett. 2019, 115, 034101. [CrossRef]

49. Karg, T.M.; Gouraud, B.; Ngai, C.T.; Schmid, G.L.; Hammerer, K.; Treutlein, P. Light-Mediated Strong Coupling between a Mechanical Oscillator and Atomic Spins 1 Meter Apart. Science 2020, 369, 174-179. [CrossRef] [PubMed]

50. Thomas, R.A.; Parniak, M.; Østfeldt, C.; Møller, C.B.; Bærentsen, C.; Tsaturyan, Y.; Schliesser, A.; Appel, J.; Zeuthen, E.; Polzik, E.S. Entanglement between Distant Macroscopic Mechanical and Spin Systems. Nat. Phys. 2020, 1-6. [CrossRef]

51. Salakhutdinov, V.; Sondermann, M.; Carbone, L.; Giacobino, E.; Bramati, A.; Leuchs, G. Single Photons Emitted by Nanocrystals Optically Trapped in a Deep Parabolic Mirror. Phys. Rev. Lett. 2020, 124, 013607. [CrossRef] [PubMed]

52. Rahman, A.T.M.A.; Barker, P.F. Laser Refrigeration, Alignment and Rotation of Levitated $\mathrm{Yb}^{3+}$ :YLF Nanocrystals. Nat. Photonics 2017, 11, 634. [CrossRef]

53. Pettit, R.M.; Neukirch, L.P.; Zhang, Y.; Vamivakas, A.N. Coherent Control of a Single Nitrogen-Vacancy Center Spin in Optically Levitated Nanodiamond. J. Opt. Soc. Am. B JOSAB 2017, 34, C31-C35. [CrossRef]

54. Delord, T.; Nicolas, L.; Schwab, L.; Hétet, G. Electron Spin Resonance from NV Centers in Diamonds Levitating in an Ion Trap. New J. Phys. 2017, 19, 033031. [CrossRef]

55. Hoang, T.M.; Ahn, J.; Bang, J.; Li, T. Electron Spin Control of Optically Levitated Nanodiamonds in Vacuum. Nat. Commun. 2016, 7, 12250. [CrossRef] [PubMed]

56. Lee, D.; Lee, K.W.; Cady, J.V.; Ovartchaiyapong, P.; Jayich, A.C.B. Topical review: Spins and mechanics in diamond. J. Opt. 2017, 19, 033001. [CrossRef]

57. Ge, L.; Zhao, N. Torsional cooling of a nanodiamond via the interaction with the electron spin of the embedded nitrogen-vacancy center. Phys. Rev. A 2018, 98, 043415. [CrossRef] 
58. Ovartchaiyapong, P.; Lee, K.W.; Myers, B.A.; Jayich, A.C.B. Dynamic strain-mediated coupling of a single diamond spin to a mechanical resonator. Nat. Commun. 2014, 5, 4429. [CrossRef] [PubMed]

59. Teissier, J.; Barfuss, A.; Appel, P.; Neu, E.; Maletinsky, P. Strain Coupling of a Nitrogen-Vacancy Center Spin to a Diamond Mechanical Oscillator. Phys. Rev. Lett. 2014, 113, 020503. [CrossRef]

60. Rondin, L.; Tetienne, J.P.; Hingant, T.; Roch, J.F.; Maletinsky, P.; Jacques, V. Magnetometry with Nitrogen-Vacancy Defects in Diamond. Rep. Prog. Phys. 2014, 77, 056503. [CrossRef]

61. Doherty, M.W.; Manson, N.B.; Delaney, P.; Jelezko, F.; Wrachtrup, J.; Hollenberg, L.C. The nitrogen-vacancy colour centre in diamond. Phys. Rep. 2013, 528, 1-45. [CrossRef]

62. Kuhlicke, A.; Schell, A.W.; Zoll, J.; Benson, O. Nitrogen vacancy center fluorescence from a submicron diamond cluster levitated in a linear quadrupole ion trap. Appl. Phys. Lett. 2014, 105, 073101. [CrossRef]

63. Neukirch, L.P.; von Haartman, E.; Rosenholm, J.M.; Vamivakas, A.N. Multi-Dimensional Single-Spin Nano-Optomechanics with a Levitated Nanodiamond. Nat. Photon. 2015, 9, 653-657. [CrossRef]

64. Hsu, J.F.; Ji, P.; Lewandowski, C.W.; D’Urso, B. Cooling the Motion of Diamond Nanocrystals in a Magneto-Gravitational Trap in High Vacuum. Sci. Rep. 2016, 6, 30125. [CrossRef] [PubMed]

65. Neukirch, L.P.; Gieseler, J.; Quidant, R.; Novotny, L.; Nick Vamivakas, A. Observation of Nitrogen Vacancy Photoluminescence from an Optically Levitated Nanodiamond. Opt. Lett. 2013, 38, 2976-2979. [CrossRef]

66. Jarmola, A.; Acosta, V.M.; Jensen, K.; Chemerisov, S.; Budker, D. Temperature- and Magnetic-Field-Dependent Longitudinal Spin Relaxation in Nitrogen-Vacancy Ensembles in Diamond. Phys. Rev. Lett. 2012, 108, 197601. [CrossRef]

67. Mittiga, T.; Hsieh, S.; Zu, C.; Kobrin, B.; Machado, F.; Bhattacharyya, P.; Rui, N.Z.; Jarmola, A.; Choi, S.; Budker, D.; et al. Imaging the Local Charge Environment of Nitrogen-Vacancy Centers in Diamond. Phys. Rev. Lett. 2018, 121, 246402. [CrossRef]

68. Tetienne, J.P.; Rondin, L.; Spinicelli, P.; Chipaux, M.; Debuisschert, T.; Roch, J.F.; Jacques, V. Magnetic-Field-Dependent Photodynamics of Single NV Defects in Diamond: An Application to Qualitative All-Optical Magnetic Imaging. New J. Phys. 2012, 14, 103033. [CrossRef]

69. Bauch, E.; Singh, S.; Lee, J.; Hart, C.A.; Schloss, J.M.; Turner, M.J.; Barry, J.F.; Pham, L.M.; Bar-Gill, N.; Yelin, S.F.; et al. Decoherence of ensembles of nitrogen-vacancy centers in diamond. Phys. Rev. B 2020, 102, 134210. [CrossRef]

70. Huillery, P.; Delord, T.; Nicolas, L.; Van Den Bossche, M.; Perdriat, M.; Hétet, G. Spin mechanics with levitating ferromagnetic particles. Phys. Rev. B 2020, 101, 134415. [CrossRef]

71. Bachelard, R.; Piovella, N.; Courteille, P.W. Cooperative scattering and radiation pressure force in dense atomic clouds. Phys. Rev. A 2011, 84, 013821. [CrossRef]

72. Panat, P.V.; Lawande, S.V. Cooperative effects on optical forces-Dicke's bullet. Int. J. Mod. Phys. B 2002, 16, 3787-3795. [CrossRef]

73. Prasanna Venkatesh, B.; Juan, M.L.; Romero-Isart, O. Cooperative Effects in Closely Packed Quantum Emitters with Collective Dephasing. Phys. Rev. Lett. 2018, 120, 033602. [CrossRef]

74. Juan, M.L.; Bradac, C.; Besga, B.; Johnsson, M.; Brennen, G.; Molina-Terriza, G.; Volz, T. Cooperatively enhanced dipole forces from artificial atoms in trapped nanodiamonds. Nat. Phys. 2017, 13, 241-245. [CrossRef]

75. Schuetz, M.J.A.; Giedke, G.; Vandersypen, L.M.K.; Cirac, J.I. High-fidelity hot gates for generic spin-resonator systems. Phys. Rev. A 2017, 95, 052335. [CrossRef]

76. Martinetz, L.; Hornberger, K.; Millen, J.; Kim, M.S.; Stickler, B.A. Quantum electromechanics with levitated nanoparticles. NPJ Quantum Inf. 2020, 6, 101. [CrossRef]

77. Arita, Y.; Simpson, S.H.; Zemánek, P.; Dholakia, K. Coherent Oscillations of a Levitated Birefringent Microsphere in Vacuum Driven by Nonconservative Rotation-Translation Coupling. Sci. Adv. 2020, 6, eaaz9858. [CrossRef] [PubMed]

78. Trojek, J.; Chvátal, L.; Zemánek, P. Optical Alignment and Confinement of an Ellipsoidal Nanorod in Optical Tweezers: A Theoretical Study. J. Opt. Soc. Am. A JOSAA 2012, 29, 1224-1236. [CrossRef]

79. Rusconi, C.C.; Romero-Isart, O. Magnetic rigid rotor in the quantum regime: Theoretical toolbox. Phys. Rev. B 2016, $93,054427$. [CrossRef]

80. Jackson Kimball, D.F.; Sushkov, A.O.; Budker, D. Precessing Ferromagnetic Needle Magnetometer. Phys. Rev. Lett. 2016, 116, 190801. [CrossRef]

81. Rusconi, C.C.; Pöchhacker, V.; Kustura, K.; Cirac, J.I.; Romero-Isart, O. Quantum Spin Stabilized Magnetic Levitation. Phys. Rev. Lett. 2017, 119, 167202. [CrossRef]

82. Perdriat, M.; Huillery, P.; Pellet-Mary, C.; Hétet, G. Angle Locking of a Levitating Diamond using Spin-Diamagnetism. arXiv 2021, arXiv:2102.13637.

83. Pellet-Mary, C.; Huillery, P.; Perdriat, M.; Hétet, G. Magnetic-Torque Enhanced by Tunable Dipolar interactions. arXiv 2021, arXiv:2103.00836.

84. Horowitz, V.R.; Alemán, B.J.; Christle, D.J.; Cleland, A.N.; Awschalom, D.D. Electron Spin Resonance of Nitrogen-Vacancy Centers in Optically Trapped Nanodiamonds. Proc. Natl. Acad. Sci. USA 2012, 109, 13493-13497. [CrossRef]

85. Wood, A.A.; Lilette, E.; Fein, Y.Y.; Tomek, N.; McGuinness, L.P.; Hollenberg, L.C.L.; Scholten, R.E.; Martin, A.M. Quantum measurement of a rapidly rotating spin qubit in diamond. Sci. Adv. 2018, 4. [CrossRef] [PubMed]

86. Delord, T.; Huillery, P.; Schwab, L.; Nicolas, L.; Lecordier, L.; Hétet, G. Ramsey Interferences and Spin Echoes from Electron Spins Inside a Levitating Macroscopic Particle. Phys. Rev. Lett. 2018, 121, 053602. [CrossRef] 
87. Geiselmann, M.; Juan, M.L.; Renger, J.; Say, J.M.; Brown, L.J.; de Abajo, F.J.G.; Koppens, F.; Quidant, R. Three-dimensional optical manipulation of a single electron spin. Nat. Nanotechnol. 2013, 8, 175-179. [CrossRef] [PubMed]

88. van der Laan, F.; Reimann, R.; Tebbenjohanns, F.; Vijayan, J.; Novotny, L.; Frimmer, M. Observation of radiation torque shot noise on an optically levitated nanodumbbell. arXiv 2020, arXiv:2012.14231

89. Jain, V.; Gieseler, J.; Moritz, C.; Dellago, C.; Quidant, R.; Novotny, L. Direct Measurement of Photon Recoil from a Levitated Nanoparticle. Phys. Rev. Lett. 2016, 116, 243601. [CrossRef]

90. Kim, P.H.; Hauer, B.D.; Doolin, C.; Souris, F.; Davis, J.P. Approaching the standard quantum limit of mechanical torque sensing. Nat. Commun. 2016, 7, 13165. [CrossRef] [PubMed]

91. Ahn, J.; Xu, Z.; Bang, J.; Ju, P.; Gao, X.; Li, T. Ultrasensitive torque detection with an optically levitated nanorotor. Nat. Nanotechnol. 2020, 15, 89-93. [CrossRef]

92. Hempston, D.; Vovrosh, J.; Toroš, M.; Winstone, G.; Rashid, M.; Ulbricht, H. Force sensing with an optically levitated charged nanoparticle. Appl. Phys. Lett. 2017, 111, 133111. [CrossRef]

93. Ranjit, G.; Cunningham, M.; Casey, K.; Geraci, A.A. Zeptonewton force sensing with nanospheres in an optical lattice. Phys. Rev. A 2016, 93, 053801. [CrossRef]

94. Prat-Camps, J.; Teo, C.; Rusconi, C.C.; Wieczorek, W.; Romero-Isart, O. Ultrasensitive Inertial and Force Sensors with Diamagnetically Levitated Magnets. Phys. Rev. Appl. 2017, 8, 034002. [CrossRef]

95. Fuchs, G.; Dobrovitski, V.; Toyli, D.; Heremans, F.; Awschalom, D. Gigahertz dynamics of a strongly driven single quantum spin. Science 2009, 326, 1520-1522. [CrossRef]

96. Rugar, D.; Budakian, R.; Mamin, H.J.; Chui, B.W. Single spin detection by magnetic resonance force microscopy. Nature 2004, 430, 329. [CrossRef]

97. Huillery, P.; Leibold, J.; Delord, T.; Nicolas, L.; Achard, J.; Tallaire, A.; Hétet, G. Coherent Microwave Control of a Nuclear Spin Ensemble at Room Temperature. arXiv 2020, arXiv:2005.13082

98. Frangeskou, A.C.; Rahman, A.T.M.A.; Gines, L.; Mandal, S.; Williams, O.A.; Barker, P.F.; Morley, G.W. Pure Nanodiamonds for Levitated Optomechanics in Vacuum. New J. Phys. 2018, 20, 043016. [CrossRef]

99. Chen, Y.C.; Salter, P.S.; Knauer, S.; Weng, L.; Frangeskou, A.C.; Stephen, C.J.; Ishmael, S.N.; Dolan, P.R.; Johnson, S.; Green, B.L.; et al. Laser Writing of Coherent Colour Centres in Diamond. Nat. Photon. 2017, 11, 77-80. [CrossRef]

100. Rani, D.; Opaluch, O.R.; Neu, E. Recent Advances in Single Crystal Diamond Device Fabrication for Photonics, Sensing and Nanomechanics. Micromachines 2021, 12,36. [CrossRef]

101. Kuhn, S.; Asenbaum, P.; Kosloff, A.; Sclafani, M.; Stickler, B.A.; Nimmrichter, S.; Hornberger, K.; Cheshnovsky, O.; Patolsky, F.; Arndt, M. Cavity-Assisted Manipulation of Freely Rotating Silicon Nanorods in High Vacuum. Nano Lett. 2015, 15, 5604-5608. [CrossRef]

102. Mrózek, M.; Rudnicki, D.; Kehayias, P.; Jarmola, A.; Budker, D.; Gawlik, W. Longitudinal spin relaxation in nitrogen-vacancy ensembles in diamond. EPJ Quantum Technol. 2015, 2, 22. [CrossRef]

103. Choi, J.; Choi, S.; Kucsko, G.; Maurer, P.C.; Shields, B.J.; Sumiya, H.; Onoda, S.; Isoya, J.; Demler, E.; Jelezko, F.; et al. Depolarization Dynamics in a Strongly Interacting Solid-State Spin Ensemble. Phys. Rev. Lett. 2017, 118, 093601. [CrossRef] [PubMed]

104. Delord, T.; Nicolas, L.; Bodini, M.; Hétet, G. Diamonds Levitating in a Paul Trap under Vacuum: Measurements of Laser-Induced Heating via NV Center Thermometry. Appl. Phys. Lett. 2017, 111, 013101. [CrossRef]

105. Rahman, A.T.M.A.; Frangeskou, A.C.; Kim, M.S.; Bose, S.; Morley, G.W.; Barker, P.F. Burning and graphitization of optically levitated nanodiamonds in vacuum. Sci. Rep. 2016, 6, 21633. [CrossRef] [PubMed]

106. Webster, S.; Chen, Y.; Turri, G.; Bennett, A.; Wickham, B.; Bass, M. Intrinsic and Extrinsic Absorption of Chemical Vapor Deposition Single-Crystal Diamond from the Middle Ultraviolet to the Far Infrared. J. Opt. Soc. Am. B JOSAB 2015, 32, 479-484. [CrossRef]

107. Walker, J. Optical Absorption and Luminescence in Diamond. Rep. Prog. Phys. 1979, 42, 1605-1659. [CrossRef]

108. Acosta, V.M.; Bauch, E.; Ledbetter, M.P.; Waxman, A.; Bouchard, L.S.; Budker, D. Temperature Dependence of the NitrogenVacancy Magnetic Resonance in Diamond. Phys. Rev. Lett. 2010, 104, 070801. [CrossRef]

109. Toyli, D.M.; Christle, D.J.; Alkauskas, A.; Buckley, B.B.; Van de Walle, C.G.; Awschalom, D.D. Measurement and Control of Single Nitrogen-Vacancy Center Spins above 600 K. Phys. Rev. X 2012, 2, 031001. [CrossRef]

110. Koehl, W.F.; Buckley, B.B.; Heremans, F.J.; Calusine, G.; Awschalom, D.D. Room Temperature Coherent Control of Defect Spin Qubits in Silicon Carbide. Nature 2011, 479, 84-87. [CrossRef]

111. Redjem, W.; Durand, A.; Herzig, T.; Benali, A.; Pezzagna, S.; Meijer, J.; Kuznetsov, A.Y.; Nguyen, H.S.; Cueff, S.; Gérard, J.M.; et al. Single Artificial Atoms in Silicon Emitting at Telecom Wavelengths. Nat. Electron. 2020, 3, 738-743. [CrossRef]

112. Achard, J.; Jacques, V.; Tallaire, A. Chemical Vapour Deposition Diamond Single Crystals with Nitrogen-Vacancy Centres: A Review of Material Synthesis and Technology for Quantum Sensing Applications. J. Phys. D Appl. Phys. 2020, $53,313001$. [CrossRef]

113. Bradac, C.; Gaebel, T.; Naidoo, N.; Sellars, M.J.; Twamley, J.; Brown, L.J.; Barnard, A.S.; Plakhotnik, T.; Zvyagin, A.V.; Rabeau, J.R. Observation and Control of Blinking Nitrogen-Vacancy Centres in Discrete Nanodiamonds. Nat. Nanotechnol. 2010, 5, 345-349. [CrossRef]

114. Feudis, M.D.; Tallaire, A.; Nicolas, L.; Brinza, O.; Goldner, P.; Hétet, G.; Bénédic, F.; Achard, J. Large-Scale Fabrication of Highly Emissive Nanodiamonds by Chemical Vapor Deposition with Controlled Doping by SiV and GeV Centers from a Solid Source. Adv. Mater. Interfaces 2020, 7, 1901408. [CrossRef] 
115. Tallaire, A.; Brinza, O.; De Feudis, M.; Ferrier, A.; Touati, N.; Binet, L.; Nicolas, L.; Delord, T.; Hétet, G.; Herzig, T.; et al. Synthesis of Loose Nanodiamonds Containing Nitrogen-Vacancy Centers for Magnetic and Thermal Sensing. ACS Appl. Nano Mater. 2019, 2, 5952-5962. [CrossRef]

116. Mindarava, Y.; Blinder, R.; Liu, Y.; Scheuer, J.; Lang, J.; Agafonov, V.; Davydov, V.A.; Laube, C.; Knolle, W.; Abel, B.; et al. Synthesis and Coherent Properties of 13C-Enriched Sub-Micron Diamond Particles with Nitrogen Vacancy Color Centers. Carbon 2020, 165, 395-403. [CrossRef] 